Supporting Information Figures and Tables

\title{
Lists of Chemical Warfare Agents and Precursors from International Nonproliferation Frameworks: Structural Annotation and Chemical Fingerprint Analysis
}

Stefano Costanzi, ${ }^{a}$ Charlotte K. Slavick, ${ }^{a}$ Brent O. Hutcheson, ${ }^{a}$ Gregory D. Koblentz, ${ }^{b}$ Richard T. Cupitt ${ }^{c}$

${ }^{a}$ Department of Chemistry, American University, 4400 Massachusetts Avenue, NW, Washington, DC 20016, USA

${ }^{\mathrm{b}}$ Schar School of Policy and Government, George Mason University, 3351 Fairfax Drive, Arlington, VA 22201, USA

${ }^{\mathrm{c}}$ Stimson Center, 1211 Connecticut Ave, NW, Washington, DC 20036, USA 
Figure S1. Larger format version of Figure 3. Fan plot showing the clustering of CW-control lists based on chemical structures. CWC schedule entries are shown in black, AG precursors list entries are shown in blue, and WA ML7 entries are shown in red. Large-font green labels indicate the chemical features characterizing the various clusters. Small-font green labels indicate the CWA class to which the entries comprised in the clusters belong. Subclusters within larger clusters are encased in pentagon shapes. The clusters that encompass the entries recently added to CWC schedule 1 and the AG precursors list are underlined. Outlier chemicals, i.e. chemicals that do not completely fit into the definition of the cluster to which they belong, are marked with dark red labels. 


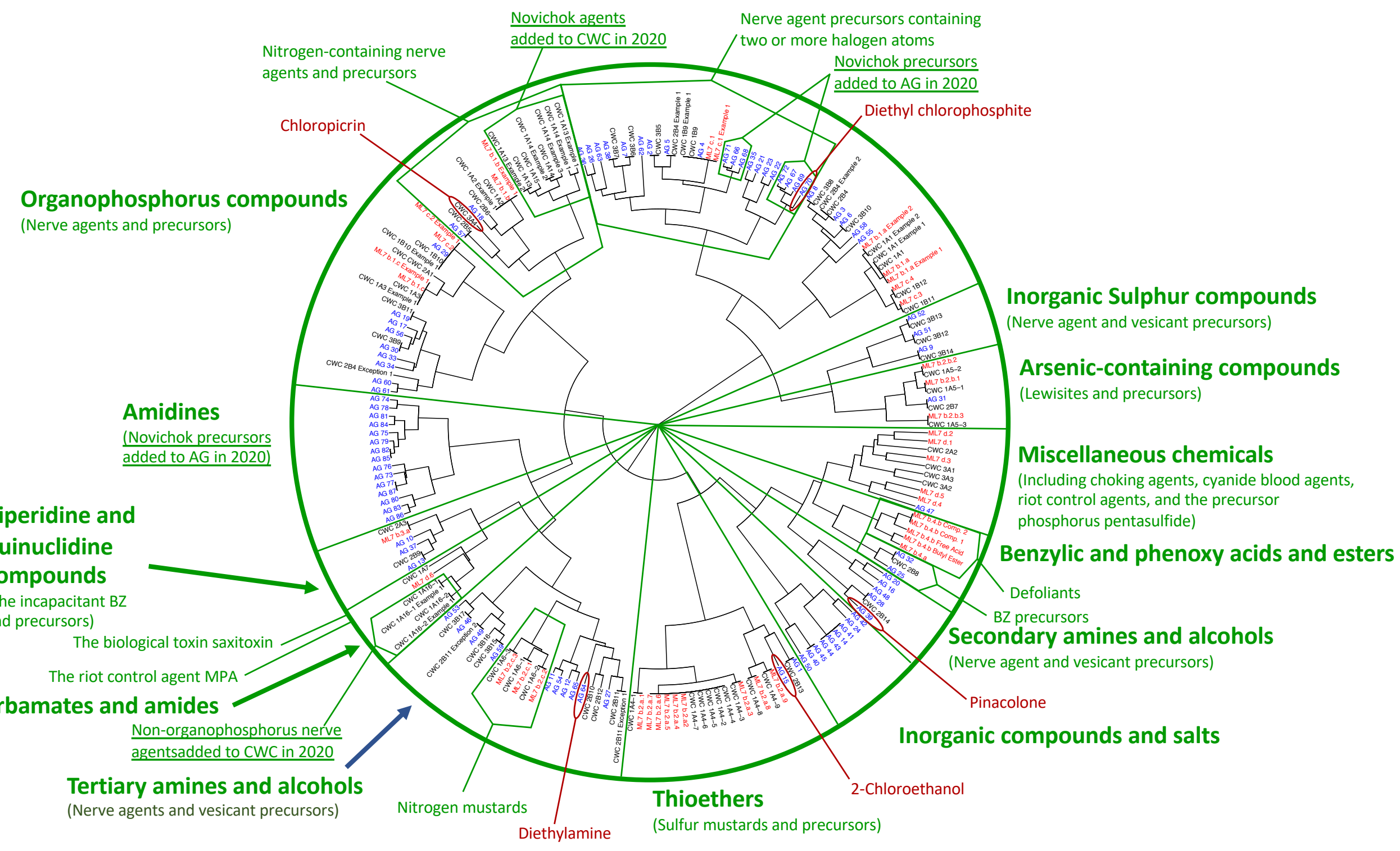


Table S1. Annotated Chemical Weapons Convention (CWC) Schedules - also available on the Costanzi Research Website at: https://costanziresearch.com/cw-nonproliferation/cw-controllists/cwc-schedules/ 


\section{Chemical Weapons Convention (CWC) Schedules}

Curated and Structurally Annotated by Costanzi Research - www.costanziresearch.com (Last Update: September 03, 2020)

The Table is available for download in the following formats: SDF, CSV, Microsoft Excel

Color Code:

Individual Chemicals

Mixtures of Chemicals

Families of

Exceptions: Compounds Belonging to a Family of Chemicals but Excluded from the CWC Schedules
Exalies of Chemicals

Disclaimer. The information in this table is provided for research and general information purposes only. The authors do not guarantee the accuracy of the information and do not take any for any consequence, including, but not limited to, financial or legal consequences, that may occur due to inaccuracies or omissions.

\section{Notes.}

1. Schedule 1 entries $1 \mathrm{~A} 13$ - $1 \mathrm{~A} 16$ entered into force on June 7, 2020

2. For entries lacking a CAS registry number, a sequential registry number assinged by Costanzi Research (CR number) is provided instead.

3. For proteins, the UNIPROT ID is provided instead.

4. Overlaps among CWC schedules, Australia Group chemicals, and Wassenaar ML 7 chemicals are provided.

5. 2D structures curated by Costanzi Research are provided as SDF and MRV files (MRV files require ChemAxon's MarvinSketch to open).

6. 3D strucures from PubChem are shown with NCBI's iCn3D. Institute of Standards and Technology, including, inter alia, analytical spectra.

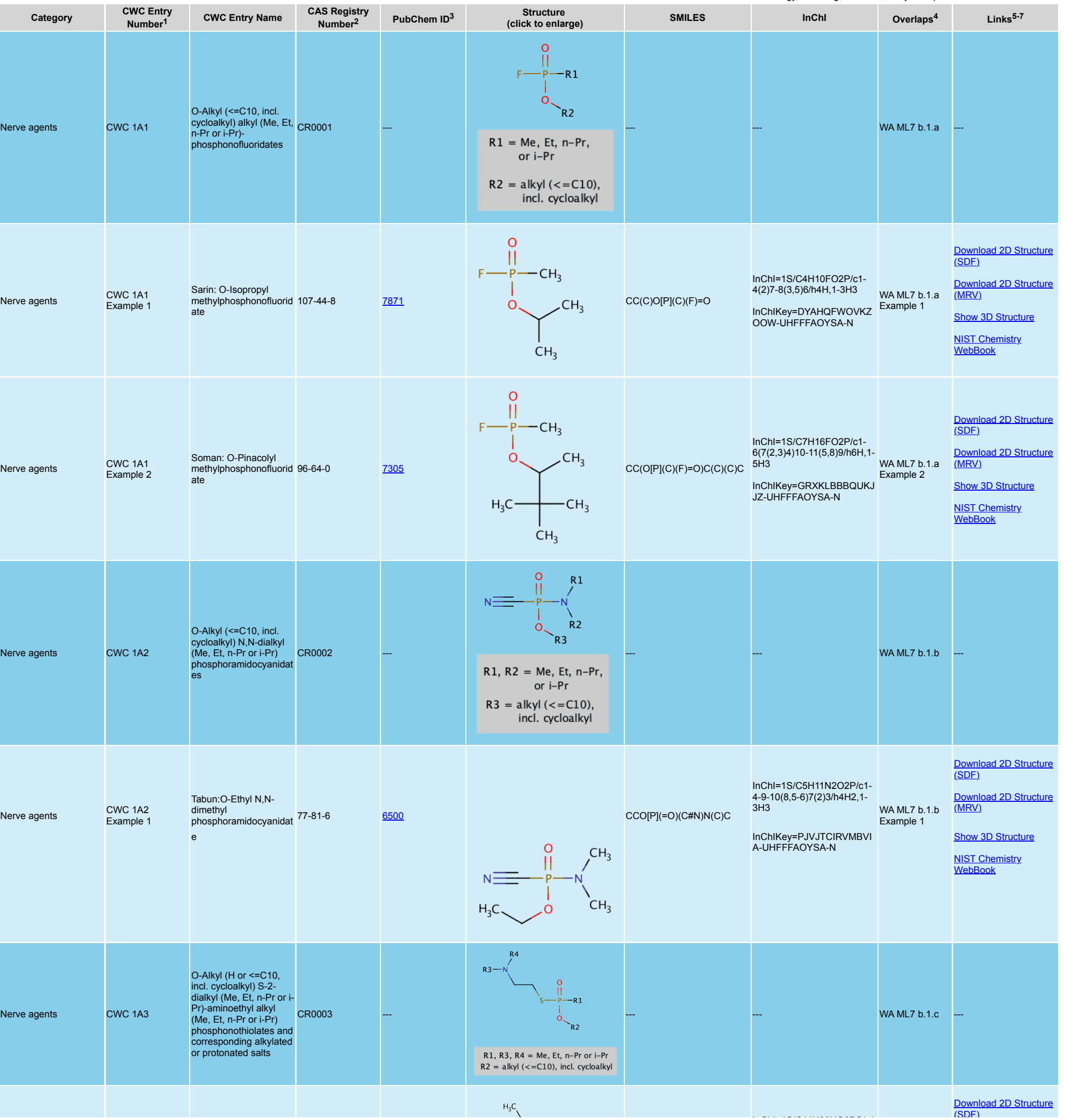




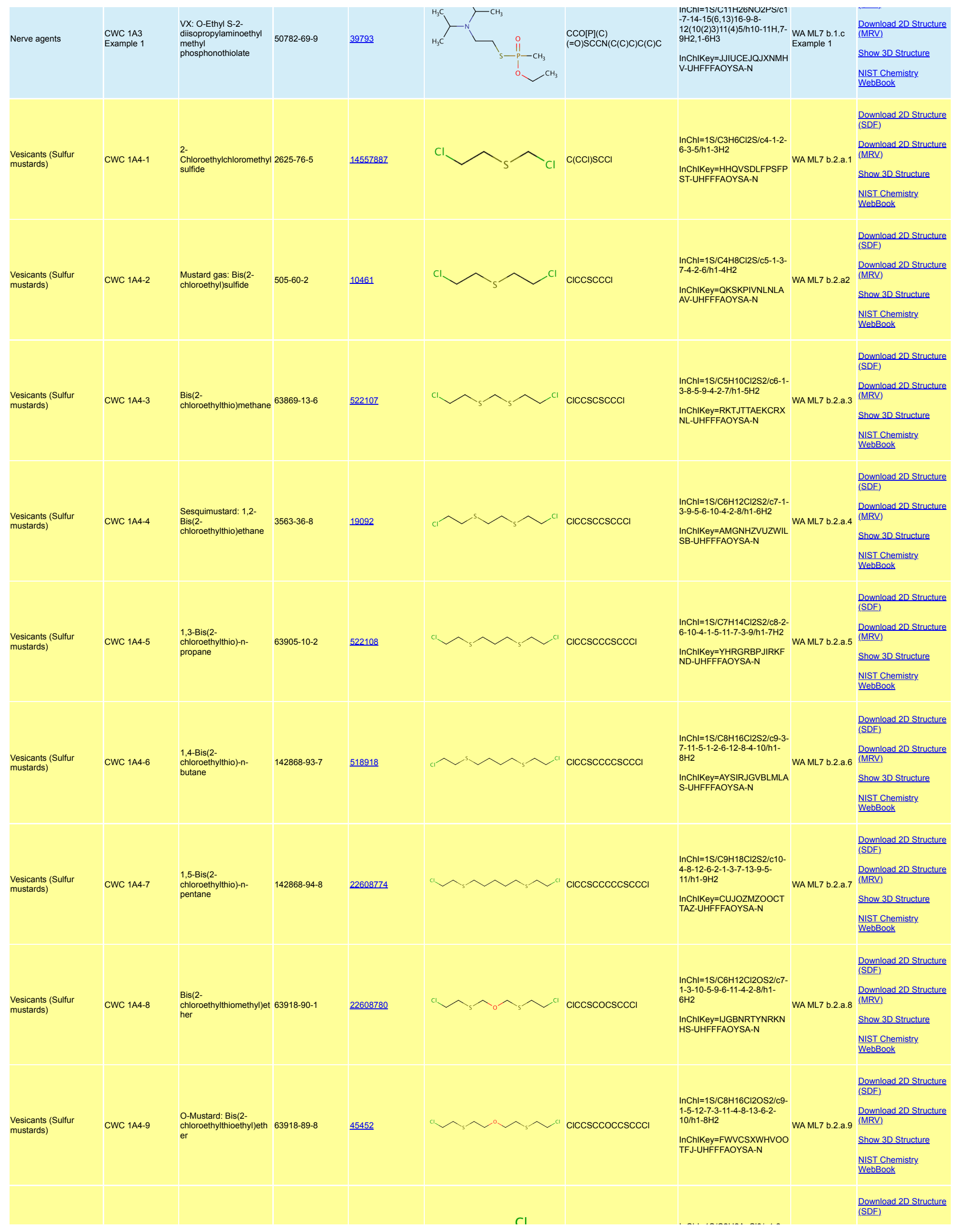




\begin{tabular}{|c|c|c|c|c|c|c|c|c|c|}
\hline Vesicants (Lewisites) & CWC 1A5-1 & $\begin{array}{l}\text { Lewisite 1: 2- } \\
\text { Chlorovinyldichloroarsin } \\
\text { e }\end{array}$ & $541-25-3$ & $\underline{5372798}$ & ${ }^{A s} \backslash \mathrm{Cl}$ & $\mathrm{Cl} / \mathrm{C}=\mathrm{C} /[\mathrm{As}](\mathrm{Cl}) \mathrm{Cl}$ & 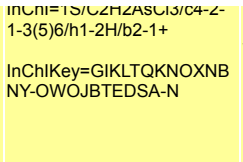 & WA ML7 b.2.b.1 & $\begin{array}{l}\text { Download 2D Structure } \\
\text { (MRV). } \\
\text { Show 3D Structure } \\
\text { NIST Chemistry. } \\
\text { WebBook }\end{array}$ \\
\hline Vesicants (Lewisites) & CWC 1A5-2 & $\begin{array}{l}\text { Lewisite 2: Bis(2- } \\
\text { chlorovinyl)chloroarsine }\end{array}$ & 40334-69-8 & $\underline{5368106}$ & & $\mathrm{Cl} / \mathrm{C}=\mathrm{C} /[\mathrm{As}](\mathrm{Cl}) / \mathrm{C}=\mathrm{C} / \mathrm{Cl}$ & $\begin{array}{l}\text { InChl=1S/C4H4AsCl3/c6-3- } \\
\text { 1-5(8)2-4-7/h1-4H/b3-1+,4- } \\
2+ \\
\text { InChIKey=YRFJGLQNTWLX } \\
\text { KO-ZPUQHVIOSA-N }\end{array}$ & WA ML7 b.2.b.2 & $\begin{array}{l}\text { Download 2D Structure } \\
\text { (SDF) } \\
\text { Download 2D Structure } \\
\text { (MRV). } \\
\text { Show 3D Structure } \\
\text { NIST Chemistry. } \\
\text { WebBook }\end{array}$ \\
\hline Vesicants (Lewisites) & CWC 1A5-3 & $\begin{array}{l}\text { Lewisite 3: Tris(2- } \\
\text { chlorovinyl)arsine }\end{array}$ & $40334-70-1$ & $\underline{5352143}$ & & $\begin{array}{l}\mathrm{Cl} / \mathrm{C}=\mathrm{C} /[\mathrm{As}] \\
(/ \mathrm{C}=\mathrm{C} / \mathrm{Cl}) / \mathrm{C}=\mathrm{C} / \mathrm{Cl}\end{array}$ & $\begin{array}{l}\text { InChl=1S/C6H6AsCl3/c8-4- } \\
\text { 1-7(2-5-9)3-6-10/h1-6H/b4- } \\
\text { 1+,5-2+,6-3+ } \\
\text { InChIKey=AOAVIJUEFJPSA } \\
\text { I-GZDDRBCLSA-N }\end{array}$ & WA ML7 b.2.b.3 & $\begin{array}{l}\text { Download 2D Structure } \\
\text { (SDF). } \\
\text { Download 2D Structure } \\
\text { (MRV). } \\
\text { Show 3D Structure } \\
\text { NIST Chemistry. } \\
\text { WebBook }\end{array}$ \\
\hline $\begin{array}{l}\text { Vesicant (Nitrogen } \\
\text { mustards) }\end{array}$ & CWC 1A6-1 & $\begin{array}{l}\text { HN1: Bis(2- } \\
\text { chloroethyl)ethylamine }\end{array}$ & $538-07-8$ & $\underline{10848}$ & & $\mathrm{CCN}(\mathrm{CCCl}) \mathrm{CCCl}$ & $\begin{array}{l}\text { InChl=1S/C6H13Cl2N/c1-2- } \\
9(5-3-7) 6-4-8 / h 2-6 \mathrm{H} 2,1 \mathrm{H} 3 \\
\\
\text { InChIKey=UQZPGHOJMQT } \\
\text { OHB-UHFFFAOYSA-N }\end{array}$ & WA ML7 b.2.c. 1 & $\begin{array}{l}\text { Download 2D Structure } \\
\text { (SDF). } \\
\text { Download 2D Structure } \\
\text { (MRV). } \\
\text { Show 3D Structure } \\
\text { NIST Chemistry. } \\
\text { WebBook }\end{array}$ \\
\hline $\begin{array}{l}\text { Vesicant (Nitrogen } \\
\text { mustards) }\end{array}$ & CWC 1A6-2 & $\begin{array}{l}\text { HN2: Bis(2- } \\
\text { chloroethyl)methylamin } \\
\text { e }\end{array}$ & $51-75-2$ & $\underline{4033}$ & & $\mathrm{CN}(\mathrm{CCCl}) \mathrm{CCCl}$ & $\begin{array}{l}\text { InChl=1S/C5H11CI2N/c1- } \\
8(4-2-6) 5-3-7 / h 2-5 \mathrm{H} 2,1 \mathrm{H} 3 \\
\\
\text { InChIKey=HAWPXGHAZFH } \\
\text { HAD-UHFFFAOYSA-N }\end{array}$ & WA ML7 b.2.c. 2 & $\begin{array}{l}\text { Download 2D Structure } \\
\text { (DF). } \\
\text { Download 2D Structure } \\
\text { (MRV). } \\
\text { Show 3D Structure } \\
\text { NIST Chemistry. } \\
\text { WebBook }\end{array}$ \\
\hline $\begin{array}{l}\text { Vesicant (Nitrogen } \\
\text { mustards) }\end{array}$ & CWC 1A6-3 & $\begin{array}{l}\text { HN3: Tris(2- } \\
\text { chloroethyl)amine }\end{array}$ & $555-77-1$ & $\underline{5561}$ & & $\mathrm{C}(\mathrm{CCl}) \mathrm{N}(\mathrm{CCCl}) \mathrm{CCCl}$ & $\begin{array}{l}\text { InChl=1S/C6H12Cl3N/c7-1- } \\
\text { 4-10(5-2-8)6-3-9/h1-6H2 } \\
\text { InChIKey=FDAYLTPAFBGX } \\
\text { AB-UHFFFAOYSA-N }\end{array}$ & WA ML7 b.2.c. 3 & $\begin{array}{l}\text { Download 2D Structure } \\
\text { ( } \text { SDF). } \\
\text { Download 2D Structure } \\
\text { (MRV). } \\
\text { Show 3D Structure } \\
\text { NIST Chemistry. } \\
\text { WebBook }\end{array}$ \\
\hline Biological toxins & CWC $1 \mathrm{~A} 7$ & Saxitoxin & $35523-89-8$ & $\underline{56947150}$ & & $\begin{array}{l}\mathrm{NC}(=\mathrm{O}) \mathrm{OC}[\mathrm{C} @ @ \mathrm{H}] 1 \mathrm{NC}(=\mathrm{N} \\
) \mathrm{N} 2 \mathrm{CCC}(\mathrm{O})(\mathrm{O}) \\
{[\mathrm{C} @ @] 22 \mathrm{~N}=\mathrm{C}(\mathrm{N}) \mathrm{N}[\mathrm{C} @ @ \mathrm{H}]} \\
12\end{array}$ & $\begin{array}{l}\text { InChl=1S/C10H17N7O4/c11- } \\
6-15-5-4(3-21-8(13) 18) 14- \\
7(12) 17-2-1- \\
9(19,20) 10(5,17) 16-6 / \mathrm{h} 4- \\
5,19-20 \mathrm{H}, 1-3 \mathrm{H} 2,(\mathrm{H} 2,12,14) \\
(\mathrm{H} 2,13,18) \\
(\mathrm{H} 3,11,15,16) / 44-, 5-, 10- \\
\text { Im0/s1 } \\
\text { InChIKey=RPQXVSUAYFXF } \\
\text { JA-HGRQIUPRSA-N }\end{array}$ & 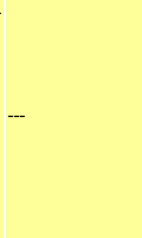 & $\begin{array}{l}\text { Download 2D Structure } \\
\text { (SDF). } \\
\text { Download 2D Structure } \\
\text { (MRV). } \\
\text { Show 3D Structure } \\
\text { NIST Chemistry. } \\
\text { WebBook }\end{array}$ \\
\hline Biological toxins & CWC 1 A 8 & Ricin & $9009-86-3$ & P02879 & & -- & --- & -- & Show 3D Structure \\
\hline $\begin{array}{l}\text { Nerve agents } \\
\text { (Novichok) }\end{array}$ & CWC $1 \mathrm{~A} 13$ & $\begin{array}{l}\text { P-alkyl }(\mathrm{H} \text { or } \leq \mathrm{C} 10 \text {, incl. } \\
\text { cycloalkyl) } \mathrm{N}-(1- \\
\text { (dialkyl( } \leq \mathrm{C} 10 \text {, incl. } \\
\text { cycloalkyl)amino))alkyli } \\
\text { dene( } \mathrm{H} \text { or } \leq \mathrm{C} 10 \text {, incl. } \\
\text { cycloalkyl) } \\
\text { phosphonamidic } \\
\text { fluorides and } \\
\text { corresponding alkylated } \\
\text { or protonated salts }\end{array}$ & CR0004 & --- & 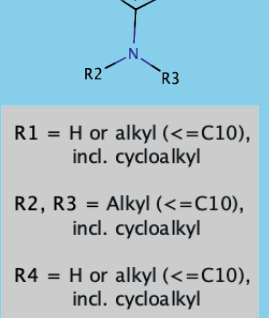 & -- & --- & --- & --- \\
\hline & & & & & & & $\begin{array}{l}\mathrm{InChl}=1 \mathrm{~S} / \mathrm{C} 40 \mathrm{H} 82 \mathrm{FN} 2 \mathrm{OP} / \mathrm{c} 1 \\
-5-9-13-17-21-25-29-33-37- \\
12 / 28-21-2 n-26-2)-18-11\end{array}$ & & $\begin{array}{l}\text { Download 2D Structure } \\
\text { (的F). }\end{array}$ \\
\hline
\end{tabular}




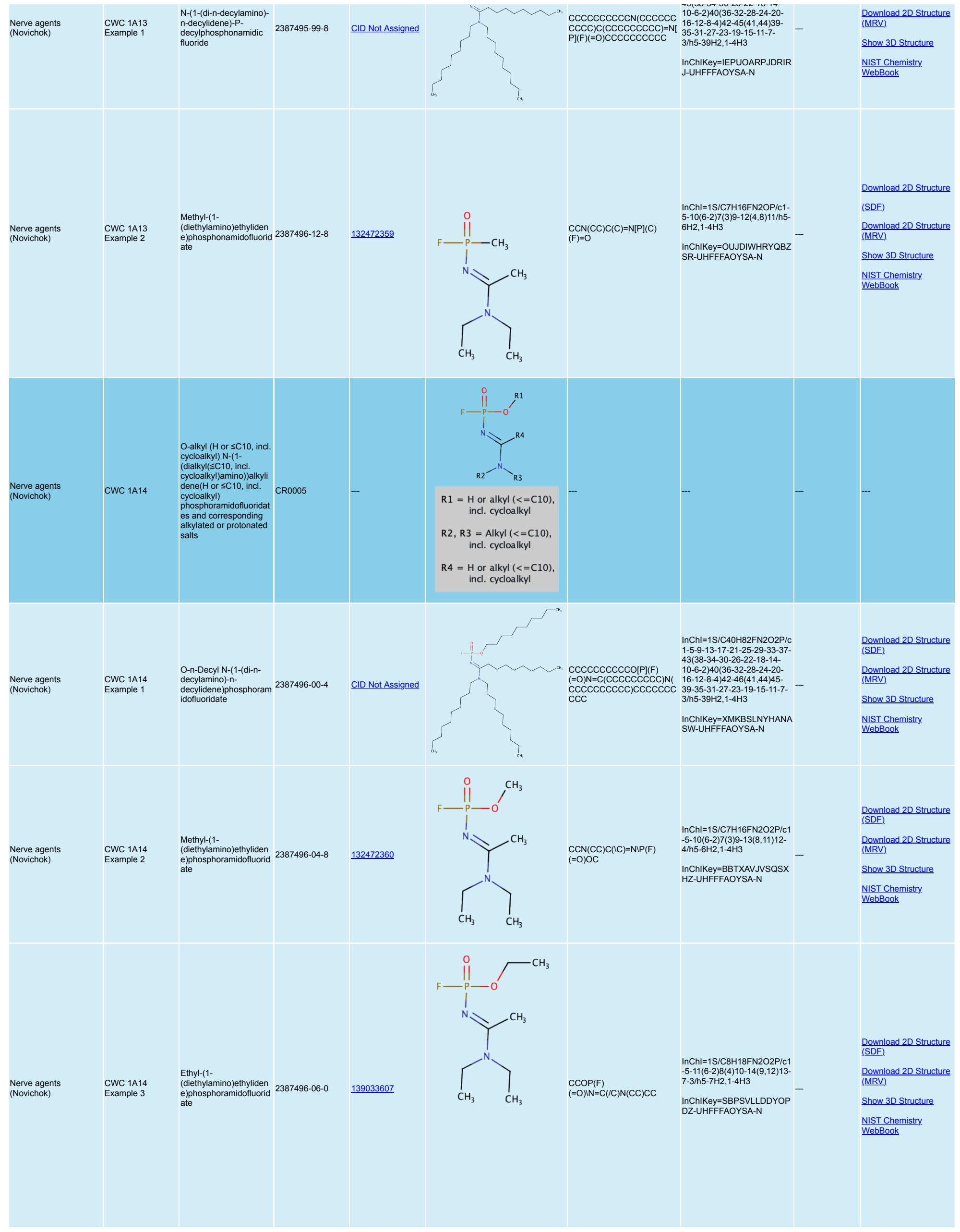




\begin{tabular}{|c|c|c|c|c|c|c|c|c|c|}
\hline $\begin{array}{l}\text { Nerve agents } \\
\text { (Novichok) }\end{array}$ & CWC 1 A15 & $\begin{array}{l}\text { Methyl- } \\
\text { (bis(diethylamino)methy } \\
\text { lene)phosphonamidoflu } \\
\text { oridate }\end{array}$ & 2387496-14-0 & $\underline{146586254}$ & CH & $\begin{array}{l}\operatorname{CCN}(C C) C(=N[P](C) \\
(F)=O) N(C C) C C\end{array}$ & $\begin{array}{l}\text { InChl=1S/C10H23FN3OP/c1 } \\
-6-13(7-2) 10(12- \\
16(5,11) 15) 14(8-3) 9-4 / h 6- \\
9 H 2,1-5 H 3 \\
\text { InChIKey=WJDIQLITBJEMB } \\
\text { A-UHFFFAOYSA-N }\end{array}$ & --- & $\begin{array}{l}\text { Download 2D Structure } \\
\text { (SDF). } \\
\text { Download 2D Structure } \\
\text { (MRV). } \\
\text { Show 3D Structure } \\
\text { NIST Chemistry. } \\
\text { WebBook }\end{array}$ \\
\hline $\begin{array}{l}\text { Nerve agents } \\
\text { (Carbamates, } \\
\text { quaternaries of } \\
\text { dimethylcarbamoyloxyp } \\
\text { yridines) }\end{array}$ & CWC 1A16-1 & $\begin{array}{l}\text { 1-[N,N-dialkyl(SC10)-N- } \\
\text { (n-(hydroxyl, cyano, } \\
\text { acetoxy)alkyl( }(\mathrm{CC} 10) \text { ) } \\
\text { ammonio]-n-[N-(3- } \\
\text { dimethylcarbamoxy- } \alpha- \\
\text { picolinyl)-N,N- } \\
\text { dialkyl( }(\leq \mathrm{C} 10) \\
\text { ammonio]decane } \\
\text { dibromide }(\mathrm{n}=1-8)\end{array}$ & CR0006 & -- & & - & -- & --- & -- \\
\hline $\begin{array}{l}\text { Nerve agents } \\
\text { (Carbamates, } \\
\text { quaternaries of } \\
\text { dimethylcarbamoyloxyp } \\
\text { yridines) }\end{array}$ & $\begin{array}{l}\text { CWC 1A16-1 } \\
\text { Example } 1\end{array}$ & $\begin{array}{l}\text { 1-[N,N-dimethyl-N-(2- } \\
\text { hydroxy)ethylammonio]- } \\
10-[\mathrm{N}-(3- \\
\text { dimethylcarbamoxy- } \alpha- \\
\text { picolinyl)-N,N- } \\
\text { dimethylammonio]deca } \\
\text { ne dibromide }\end{array}$ & $77104-62-2$ & CID Not Assigned & & 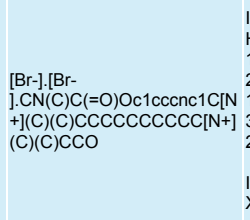 & $\begin{array}{l}\text { InChl=1S/C25H48N4O3-2Br } \\
\text { H/c1-27(2)25(31)32-24-16- } \\
15-17-26-23(24) 22- \\
29(5,6) 19-14-12-10-8-7-9- \\
11-13-18-28(3,4) 20-21- \\
\text { 30;;//h15-17,30H,7-14,18- } \\
\text { 22H2,1-6H3;2*1H/q+2;;/p-2 } \\
\text { InChIKey=SDDSMWFMJJO } \\
\text { XBZ-UHFFFAOYSA-L }\end{array}$ & --- & $\begin{array}{l}\text { Download 2D Structure } \\
\text { (SDF). } \\
\text { Download 2D Structure } \\
\text { (MRV). } \\
\text { Show 3D Structure } \\
\text { NIST Chemistry. } \\
\text { WebBook }\end{array}$ \\
\hline $\begin{array}{l}\text { Nerve agents } \\
\text { (Carbamates, } \\
\text { bisquaternaries of } \\
\text { dimethylcarbamoyloxyp } \\
\text { yridines) }\end{array}$ & CWC 1A16-2 & $\begin{array}{l}\text { 1,n-Bis[N-(3- } \\
\text { dimethylcarbamoxy- } \alpha- \\
\text { picolyl)-N,N- } \\
\text { dialkyl((sC10) } \\
\text { ammonio]-alkane- (2,(n- } \\
\text { 1)-dione) dibromide } \\
(\mathrm{n}=2-12)\end{array}$ & CR0007 & --- & & -- & -- & --- & --- \\
\hline $\begin{array}{l}\text { Nerve agents } \\
\text { (Carbamates, } \\
\text { bisquaternaries of } \\
\text { dimethylcarbamoyloxyp } \\
\text { yridines) }\end{array}$ & $\begin{array}{l}\text { CWC 1A16-2 } \\
\text { Example } 1\end{array}$ & $\begin{array}{l}\text { 1,10-Bis[ }[\mathrm{N}-(3- \\
\text { dimethylcarbamoxy- } \alpha- \\
\text { picolyl)-N-ethyl- } \mathrm{N}- \\
\text { methylammonio]decane } \\
\text {-2,9- dione dibromide }\end{array}$ & $77104-00-8$ & CID Not Assigned & & $\begin{array}{l}{[\mathrm{Br}-] .[\mathrm{Br}-] \cdot \mathrm{CC}[\mathrm{N}+](\mathrm{C})} \\
(\mathrm{CC}(=\mathrm{O}) \mathrm{CCCCCC}(=\mathrm{O}) \mathrm{C}[\mathrm{N} \\
+](\mathrm{C}) \\
(\mathrm{CC}) \mathrm{Cc} 1 \mathrm{ncccc} 1 \mathrm{OC}(=\mathrm{O}) \mathrm{N}(\mathrm{C}) \\
\mathrm{C}) \mathrm{Cc} 2 \mathrm{ncccc} 2 \mathrm{OC}(=\mathrm{O}) \mathrm{N}(\mathrm{C}) \mathrm{C}\end{array}$ & $\begin{array}{l}\text { InChl=1S/C34H54N6O6.2Br } \\
\text { H/c1-9-39(7,25-29-31(19-15- } \\
21-35-29) 45- \\
33(43) 37(3) 4) 23-27(41) 17- \\
13-11-12-14-18-28(42) 24- \\
40(8,10-2) 26-30-32(20-16- \\
22-36-30) 46- \\
34(44) 38(5) 6 ; / / / h 15-16,19- \\
22 \mathrm{H}, 9-14,17-18,23-26 \mathrm{H} 2,1- \\
8 \mathrm{H} 3 ; 2^{\star} 1 \mathrm{H} / \mathrm{q}+2 ; ; / \mathrm{p}-2 \\
\text { InChIKey=AVRROSDEBIQB } \\
\text { OJ-UHFFFAOYSA-L }\end{array}$ & --- & $\begin{array}{l}\text { Download 2D Structure } \\
\text { (SDF). } \\
\text { Download 2D Structure } \\
\text { (MRV). } \\
\text { Show 3D Structure } \\
\text { NIST Chemistry. } \\
\text { WebBook }\end{array}$ \\
\hline Precursors & CWC 1B9 & $\begin{array}{l}\text { Alkyl (Me, Et, n-Pr or i- } \\
\text { Pr) } \\
\text { phosphonyldifluorides }\end{array}$ & CR0008 & -- & $\begin{array}{l}\mathrm{R} 1=\underset{\mathrm{Pe}}{\mathrm{P}}, \mathrm{Et}, \mathrm{n}-\mathrm{Pr} \text {, } \\
\text { or } \mathrm{i}-\mathrm{Pr}\end{array}$ & -- & -- & $\begin{array}{l}\text { AG } 4,23 \\
\text { WA ML7 c. } 1\end{array}$ & --- \\
\hline Precursors & $\begin{array}{l}\text { CWC 1B9 } \\
\text { Example } 1\end{array}$ & $\begin{array}{l}\text { DF: } \\
\text { Methylphosphonyldifluo } \\
\text { ride }\end{array}$ & 676-99-3 & $\underline{69610}$ & $F-P$ & $C[P](F)(F)=0$ & $\begin{array}{l}\text { InChl=1S/CH3F2OP/c1- } \\
5(2,3) 4 / h 1 \mathrm{H} 3 \\
\text { InChIKey=PQIOSYKVBBWR } \\
\text { RI-UHFFFAOYSA-N }\end{array}$ & $\begin{array}{l}\text { AG } 4 \\
\text { WA ML7 c. } 1 \\
\text { Example } 1\end{array}$ & $\begin{array}{l}\text { Download 2D Structure } \\
\text { (SDF). } \\
\text { Download 2D Structure } \\
\text { (MRV). } \\
\text { Show 3D Structure } \\
\text { NIST Chemistry } \\
\text { WebBook }\end{array}$ \\
\hline Precursors & CWC 1B10 & $\begin{array}{l}\text { O-Alkyl }(\mathrm{H} \text { or }<=\mathrm{C} 10 \text {, } \\
\text { incl. cycloalkyl) O-2- } \\
\text { dalkyl (Me, Et, n-Pr or i- } \\
\text { Pr)-aminoethyl alkyl } \\
\text { (Me, Et, n-Pr or i-Pr) } \\
\text { phosphonites and } \\
\text { corresponding alkylated } \\
\text { or protonated salts }\end{array}$ & CR0009 & --- & $\begin{array}{l}\mathrm{R} 1, \mathrm{R} 3, \mathrm{R} 4=\mathrm{Me}, \mathrm{Et}, \mathrm{n} \text {-Pr or i-Pr } \\
\mathrm{R} 2=\text { alkyl }(<=\mathrm{C} 10) \text {, incl. cycloalkyl }\end{array}$ & -.. & -- & $\begin{array}{l}\text { AG } 29 \\
\text { WA ML7 c. } 2\end{array}$ & --- \\
\hline Precursors & $\begin{array}{l}\text { CWC } 1 \text { B10 } \\
\text { Example } 1\end{array}$ & $\begin{array}{l}\text { QL: O-Ethyl O-2- } \\
\text { diisopropylaminoethyl } \\
\text { methylphosphonite }\end{array}$ & 57856-11-8 & 170325 & $\mathrm{H}_{3} \mathrm{C}$ & $\begin{array}{l}\operatorname{CcOP}(\mathrm{C}) \operatorname{OCCN}(\mathrm{C}(\mathrm{C}) \mathrm{C}) \mathrm{C}(\mathrm{C}) \\
\mathrm{C}\end{array}$ & $\begin{array}{l}\text { InChl=1S/C11H26NO2P/c1- } \\
7-13-15(6) 14-9-8- \\
12(10(2) 3) 11(4) 5 / h 10-11 \mathrm{H}, 7- \\
9 \mathrm{H} 2,1-6 \mathrm{H} 3 \\
\text { InChIKey=OIQVKKOBTVZIF } \\
\text { E-UHFFFAOYSA-N }\end{array}$ & $\begin{array}{l}\text { AG } 29 \\
\text { WA ML7 c. } 2 \\
\text { Example } 1\end{array}$ & $\begin{array}{l}\text { Download 2D Structure } \\
\text { (SDF). } \\
\text { Download 2D Structure } \\
\text { (MRV). } \\
\text { Show 3D Structure } \\
\text { NIST Chemistry } \\
\text { WebBook }\end{array}$ \\
\hline
\end{tabular}




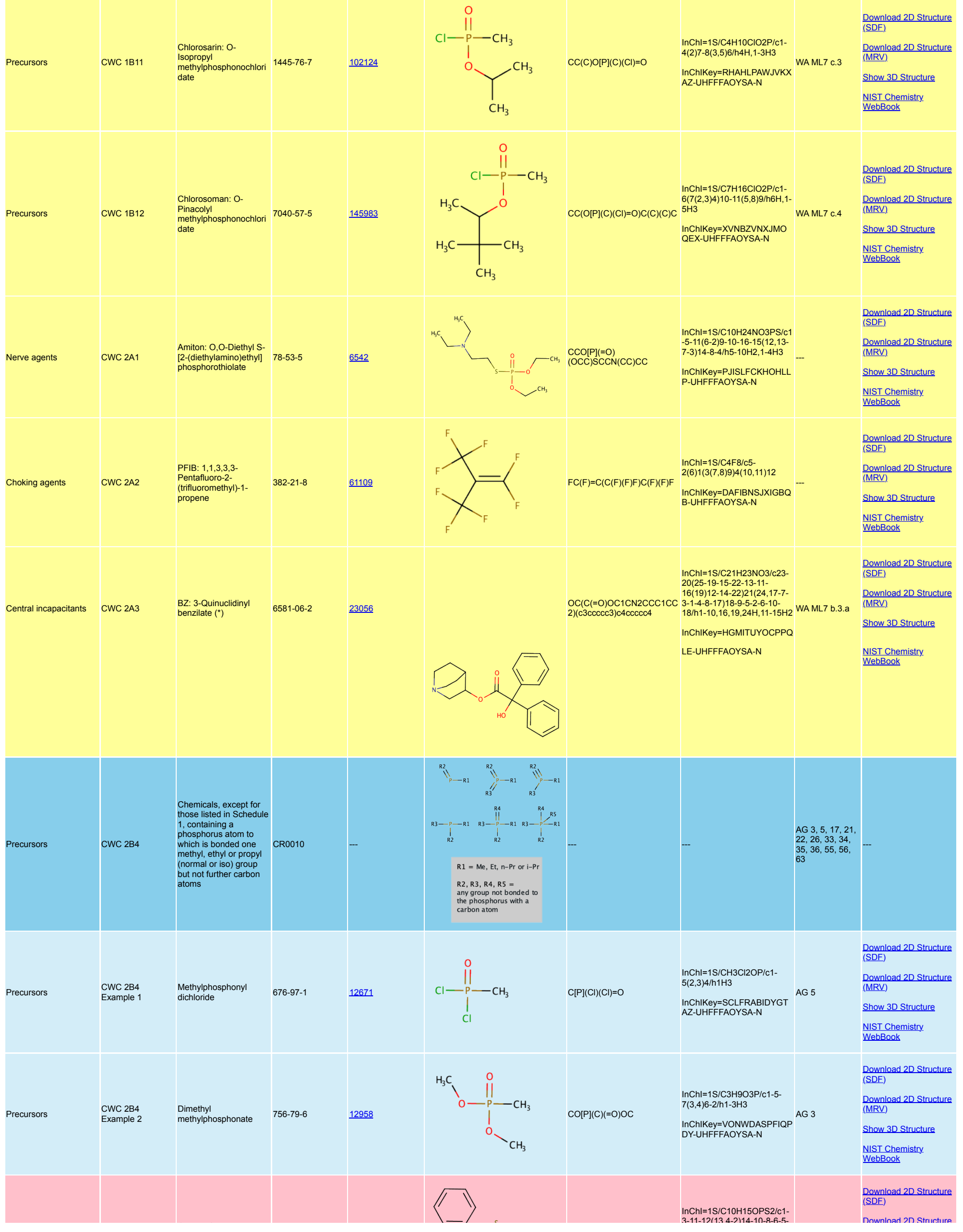




\begin{tabular}{|c|c|c|c|c|c|c|c|c|c|}
\hline Exceptions & $\begin{array}{l}\text { CWC 2B4 } \\
\text { Exception } 1\end{array}$ & $\begin{array}{l}\text { U-tthyl S-phenyl } \\
\text { ethylphosphonothiolothi } \\
\text { onate }\end{array}$ & $944-22-9$ & 13676 & 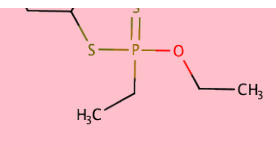 & $\mathrm{CCO}[\mathrm{P}](=\mathrm{S})(\mathrm{CC}) \mathrm{Sc} 1 \mathrm{ccccc} 1$ & $\begin{array}{l}\text { 7-9-10/h5-9H,3-4H2,1-2H3 } \\
\text { InChIKey=KVGLBTYUCJYM } \\
\text { ND-UHFFFAOYSA-N }\end{array}$ & -- & $\begin{array}{l}\text { (MRV) } \\
\text { Show 3D Structure } \\
\text { NIST Chemistry. } \\
\text { WebBook }\end{array}$ \\
\hline Precursors & CWC 2B5 & $\begin{array}{l}\text { N,N-Dialkyl (Me, Et, n- } \\
\text { Pror i-Pr) } \\
\text { phosphoramidic } \\
\text { dihalides }\end{array}$ & CR0011 & --- & $\begin{array}{l}\mathrm{R} 1, \mathrm{R} 2=\mathrm{Me}, \mathrm{Et}, \mathrm{n}-\mathrm{Pr} \text {, or i-Pr } \\
\mathrm{X}=\text { halogen }\end{array}$ & --- & -- & AG 57 & --- \\
\hline Precursors & CWC 2B6 & $\begin{array}{l}\text { Dialkyl (Me, Et, n-Pr or } \\
\text { i-Pr) N,N-dialkyl (Me, } \\
\text { Et, n-Pror i-Pr)- } \\
\text { phosphoramidates }\end{array}$ & CR0012 & --- & $\begin{array}{l}\mathrm{R} 1, \mathrm{R} 2, \mathrm{R} 3, \mathrm{R} 4=\mathrm{Me}, \mathrm{Et}, \mathrm{n}-\mathrm{Pr} \text {, or } \mathrm{i}-\mathrm{Pr} \\
\mathrm{R4}\end{array}$ & --- & --- & AG 18 & --- \\
\hline Precursors & CWC 2B7 & Arsenic trichloride & $7784-34-1$ & $\underline{24570}$ & & $\mathrm{Cl}[\mathrm{As}](\mathrm{Cl}) \mathrm{Cl}$ & $\begin{array}{l}\text { InChl=1S/AsCl3/c2-1(3)4 } \\
\text { InChlKey=OEYOHULQRFX } \\
\text { ULB-UHFFFAOYSA-N }\end{array}$ & AG 31 & $\begin{array}{l}\text { Download 2D Structure } \\
\text { (SDF) } \\
\text { Download 2D Structure } \\
\text { (MRV) } \\
\text { Show 3D Structure } \\
\text { NIST Chemistry } \\
\text { WebBook }\end{array}$ \\
\hline Precursors & CWC 2B8 & $\begin{array}{l}\text { 2,2-Diphenyl-2- } \\
\text { hydroxyacetic acid }\end{array}$ & 76-93-7 & $\underline{6463}$ & & $\begin{array}{l}\mathrm{OC}(=\mathrm{O}) \mathrm{C}(\mathrm{O}) \\
(\mathrm{c} 1 \mathrm{ccccc} 1) \mathrm{c} 2 \mathrm{ccccc} 2\end{array}$ & $\begin{array}{l}\text { InChl=1S/C14H12O3/c15- } \\
\text { 13(16)14(17,11-7-3-1-4-8- } \\
\text { 11)12-9-5-2-6-10-12/h1- } \\
\text { 10,17H,(H,15,16) } \\
\text { InChIKey=UKXSKSHDVLQN } \\
\text { KG-UHFFFAOYSA-N }\end{array}$ & AG 32 & $\begin{array}{l}\text { Download 2D Structure } \\
\text { (SDF). } \\
\text { Download 2D Structure } \\
\text { (MRV). } \\
\text { Show 3D Structure } \\
\text { NIST Chemistry. } \\
\text { WebBook }\end{array}$ \\
\hline Precursors & CWC 2B9 & Quinuclidin-3-ol & $1619-34-7$ & $\underline{15381}$ & $\mathrm{HO}$ & OC1CN2CCC1CC2 & $\begin{array}{l}\text { InChl=1S/C7H13NO/c9-7-5- } \\
8-3-1-6(7) 2-4-8 / h 6-7,9 H, 1- \\
5 \mathrm{H} 2 \\
\text { InChIKey=IVLICPVPXWEG } \\
\text { CA-UHFFFAOYSA-N }\end{array}$ & AG 13 & $\begin{array}{l}\text { Download 2D Structure } \\
\text { (SDF). } \\
\text { Download 2D Structure } \\
\text { (MRV). } \\
\text { Show 3D Structure } \\
\text { NIST Chemistry } \\
\text { WebBook }\end{array}$ \\
\hline Precursors & CWC 2B10 & $\begin{array}{l}\text { N,N-Dialkyl (Me, Et, n- } \\
\text { Pr or i-Pr) aminoethyl-2- } \\
\text { chlorides and } \\
\text { corresponding } \\
\text { protonated salts }\end{array}$ & CR0013 & --- & $\mathrm{R} 1, \mathrm{R} 2=\mathrm{Cl}$ & --- & --- & AG 11, 54 & --. \\
\hline Precursors & CWC 2B11 & $\begin{array}{l}\text { N,N-Dialkyl (Me, Et, } n- \\
\text { Pr or i-Pr) aminoethane- } \\
\text { 2-ols and corresponding } \\
\text { protonated salts }\end{array}$ & CR0014 & --- & $\mathrm{R} 1, \mathrm{R} 2=\mathrm{Me}, \mathrm{Et}, \mathrm{n}-\mathrm{Pr}$ or $\mathrm{i}-\mathrm{Pr}$ & --. & --- & AG 27 & -.- \\
\hline Exceptions & $\begin{array}{l}\text { CWC 2B11 } \\
\text { Exception } 1\end{array}$ & $\begin{array}{l}\mathrm{N}, \mathrm{N}- \\
\text { Dimethylaminoethanol } \\
\text { and corresponding } \\
\text { protonated salts }\end{array}$ & $108-01-0$ & 7902 & & $\mathrm{CN}(\mathrm{C}) \mathrm{CCO}$ & $\begin{array}{l}\text { InChl=1S/C4H11 NO/c1- } \\
5(2) 3-4-6 / h 6 \mathrm{H}, 3-4 \mathrm{H} 2,1-2 \mathrm{H} 3 \\
\text { InChIKey=UEEJHVSXFDXP } \\
\text { FK-UHFFFAOYSA-N }\end{array}$ & --- & $\begin{array}{l}\text { Download 2D Structure } \\
\text { (SDF). } \\
\text { Download 2D Structure } \\
\text { (MRV). } \\
\text { Show 3D Structure } \\
\text { NIST Chemistry. } \\
\text { WebBook }\end{array}$ \\
\hline Exceptions & $\begin{array}{l}\text { CWC 2B11 } \\
\text { Exception } 2\end{array}$ & $\begin{array}{l}\mathrm{N}, \mathrm{N}- \\
\text { Diethylaminoethanol } \\
\text { and corresponding } \\
\text { protonated salts }\end{array}$ & $100-37-8$ & $\underline{7497}$ & $\mathrm{H}_{3} \mathrm{C}$ & $\mathrm{CCN}(\mathrm{CC}) \mathrm{CCO}$ & $\begin{array}{l}\text { InChl=1S/C6H15NO/c1-3- } \\
7(4-2) 5-6-8 / h 8 H, 3-6 \mathrm{H} 2,1- \\
2 \mathrm{H} 3 \\
\text { InChIKey=BFSVOASYOCH } \\
\text { EOV-UHFFFAOYSA-N }\end{array}$ & AG 49 & $\begin{array}{l}\text { Download 2D Structure } \\
\text { (SDF). } \\
\text { Download 2D Structure } \\
\text { (MRV). } \\
\text { Show 3D Structure } \\
\text { NIST Chemistry. } \\
\text { WebBook }\end{array}$ \\
\hline Precursors & CWC 2B12 & $\begin{array}{l}\mathrm{N}, \mathrm{N} \text {-Dialkyl (Me, Et, } \mathrm{n} \text { - } \\
\text { Pror i-Pr) aminoethane- } \\
\text { 2-thiols and } \\
\text { corresponding } \\
\text { protonated salts }\end{array}$ & CR0015 & --- & $R 1, R 2=M e, E t, n-P r$ or $i-P r$ & -- & --- & AG 12,65 & --- \\
\hline
\end{tabular}




\begin{tabular}{|c|c|c|c|c|c|c|c|c|c|}
\hline Precursors & CWC 2B13 & $\begin{array}{l}\text { Thiodiglycol: Bis(2- } \\
\text { hydroxyethyl)sulfide }\end{array}$ & $111-48-8$ & 5447 & HO & occscco & $\begin{array}{l}\text { InChl=1S/C4H10O2S/c5-1- } \\
\text { 3-7-4-2-6/h5-6H,1-4H2 } \\
\text { InChIKey=YODZTKMDCQEE } \\
\text { PHD-UHFFFAOYSA-N }\end{array}$ & AG 1 & $\begin{array}{l}\text { Download 2D Structure } \\
\text { (MRV). } \\
\text { Show 3D Structure } \\
\text { NIST Chemistry. } \\
\text { WebBook }\end{array}$ \\
\hline Precursors & CWC 2B14 & $\begin{array}{l}\text { Pinacolyl alcohol: 3,3- } \\
\text { Dimethylbutan-2-ol }\end{array}$ & $464-07-3$ & 10045 & $\left.\mathrm{H}_{3} \mathrm{C}\right|^{\mathrm{CH}_{3}} \mathrm{CH}_{3}$ & $\mathrm{CC}(\mathrm{O}) \mathrm{C}(\mathrm{C})(\mathrm{C}) \mathrm{C}$ & $\begin{array}{l}\text { InChl=1S/C6H14O/c1- } \\
5(7) 6(2,3) 4 / h 5,7 \mathrm{H}, 1-4 \mathrm{H} 3 \\
\text { InChlKey=DFOXKPDFWGN } \\
\text { LJU-UHFFFAOYSA-N }\end{array}$ & AG 28 & $\begin{array}{l}\text { Download 2D Structure } \\
\text { (SDF). } \\
\text { Download 2D Structure } \\
\text { (MRV). } \\
\text { Show 3D Structure } \\
\text { NIST Chemistry. } \\
\text { WebBook }\end{array}$ \\
\hline Choking agents & CWC 3A1 & $\begin{array}{l}\text { Phosgene: Carbonyl } \\
\text { dichloride }\end{array}$ & $75-44-5$ & 6371 & & $C I C(C I)=0$ & $\begin{array}{l}\text { InChl=1S/CCI2O/c2-1(3)4 } \\
\text { InChIKey=YGYAWVDWMAB } \\
\text { LBF-UHFFFAOYSA-N }\end{array}$ & -- & $\begin{array}{l}\text { Download 2D Structure } \\
\text { (SDF) } \\
\text { Download 2D Structure } \\
\text { (MRV). } \\
\text { Show 3D Structure } \\
\text { NIST Chemistry. } \\
\text { WebBook }\end{array}$ \\
\hline Blood agents & CWC 3 A2 & Cyanogen chloride & $506-77-4$ & $\underline{10477}$ & $\mathrm{Cl}=$ & CIC\#N & $\begin{array}{l}\text { InChl=1S/CCIN/c2-1-3 } \\
\text { InChIKey=QPJDMGCKMHU } \\
\text { XFD-UHFFFAOYSA-N }\end{array}$ & -- & $\begin{array}{l}\text { Download 2D Structure } \\
\text { (SDF). } \\
\text { Download 2D Structure } \\
\text { (MRV). } \\
\text { Show 3D Structure } \\
\text { NIST Chemistry. } \\
\text { WebBook }\end{array}$ \\
\hline Blood agents & CWC 3 A 3 & Hydrogen cyanide & $74-90-8$ & $\underline{768}$ & $\mathrm{HC} \equiv \mathrm{N}$ & $\mathrm{C \# N}$ & $\begin{array}{l}\text { InChl=1S/CHN/c1-2/h1H } \\
\text { InChIKey=LELOWRISYMNN } \\
\text { SU-UHFFFAOYSA-N }\end{array}$ & 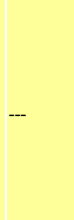 & $\begin{array}{l}\frac{\text { Download 2D Structure }}{(\text { SDF) }} \\
\text { Download 2D Structure } \\
\text { (MRV). } \\
\text { Show 3D Structure } \\
\text { NIST Chemistry. } \\
\text { WebBook }\end{array}$ \\
\hline Choking agents & CWC 3A4 & $\begin{array}{l}\text { Chloropicrin: } \\
\text { Trichloronitromethane }\end{array}$ & $76-06-2$ & $\underline{6423}$ & $\left.\right|_{\mathrm{Cl}}$ & {$\left[\mathrm{O}-\left[\mathrm{N}^{+}\right](=\mathrm{O}) \mathrm{C}(\mathrm{Cl})(\mathrm{Cl}) \mathrm{Cl}\right.$} & $\begin{array}{l}\text { InChl=1S/CCI3NO2/c2- } \\
1(3,4) 5(6) 7 \\
\text { InChIKey=LFHISGNCFUNF } \\
\text { FM-UHFFFAOYSA-N }\end{array}$ & -- & $\begin{array}{l}\frac{\text { Download 2D Structure }}{\text { (SDF). }} \\
\text { Download 2D Structure } \\
\text { (MRV). } \\
\text { Show 3D Structure } \\
\text { NIST Chemistry. } \\
\text { WebBook }\end{array}$ \\
\hline Precursors & CWC 3B5 & Phosphorus oxychloride & $10025-87-3$ & $\underline{24813}$ & $\mathrm{Cl}-\prod_{\mathrm{P}-}^{\mathrm{Cl}}$ & $\mathrm{Cl}[\mathrm{P}](\mathrm{Cl})(\mathrm{Cl})=\mathrm{O}$ & $\begin{array}{l}\text { InChl=1S/CI3OP/c1-5(2,3)4 } \\
\text { InChIKey=XHXXXVLFKHQF } \\
\text { AL-UHFFFAOYSA-N }\end{array}$ & $A G 2$ & $\begin{array}{l}\text { Download 2D Structure } \\
\text { (SDF). } \\
\text { Download 2D Structure } \\
\text { (MRV). } \\
\text { Show 3D Structure } \\
\text { NIST Chemistry. } \\
\text { WebBook }\end{array}$ \\
\hline Precursors & CWC 3B6 & Phosphorus trichloride & 7719-12-2 & $\underline{24387}$ & & $\mathrm{ClP}(\mathrm{Cl}) \mathrm{Cl}$ & $\begin{array}{l}\text { InChl=1S/CI3P/c1-4(2)3 } \\
\text { InChIKey=FAIAAWCVCHQX } \\
\text { DN-UHFFFAOYSA-N }\end{array}$ & AG 7 & $\begin{array}{l}\frac{\text { Download 2D Structure }}{\text { (SDF). }} \\
\text { Download 2D Structure } \\
\text { (MRV). } \\
\text { Show 3D Structure } \\
\text { NIST Chemistry. } \\
\text { WebBook }\end{array}$ \\
\hline Precursors & CWC 3B7 & $\begin{array}{l}\text { Phosphorus } \\
\text { pentachloride }\end{array}$ & $10026-13-8$ & $\underline{24819}$ & $\mathrm{Cl}$ & $\mathrm{Cl}[\mathrm{P}](\mathrm{Cl})(\mathrm{Cl})(\mathrm{Cl}) \mathrm{Cl}$ & $\begin{array}{l}\text { InChl=1S/CI5P/C1-6(2,3,4)5 } \\
\text { InChIKey=UHZYTMXLRWX } \\
\text { GPK-UHFFFAOYSA-N }\end{array}$ & AG 38 & $\begin{array}{l}\text { Download 2D Structure } \\
\text { (SDF). } \\
\text { Download 2D Structure } \\
\text { (MRV). } \\
\text { Show 3D Structure } \\
\text { NIST Chemistry. } \\
\text { WebBook }\end{array}$ \\
\hline Precursors & CWC 3B8 & Trimethyl phosphite & $121-45-9$ & 8472 & ${ }_{\mathrm{CH}_{3}}^{\mathrm{O}}$ & $\mathrm{COP}(\mathrm{OC}) \mathrm{OC}$ & $\begin{array}{l}\text { InChl=1S/C3H9O3P/c1-4- } \\
\text { 7(5-2)6-3/h1-3H3 } \\
\text { InChIKey=CYTQBVOFDCP } \\
\text { GCX-UHFFFAOYSA-N }\end{array}$ & AG 8 & $\begin{array}{l}\text { Download 2D Structure } \\
\text { (SDF). } \\
\text { Download 2D Structure } \\
\text { (MRV). } \\
\text { Show 3D Structure } \\
\text { NIST Chemistry. } \\
\text { WebBook }\end{array}$ \\
\hline
\end{tabular}




\begin{tabular}{|c|c|c|c|c|c|c|c|c|c|}
\hline Precursors & CWC 3B9 & Triethyl phosphite & $122-52-1$ & $\underline{31215}$ & $\mathrm{CH}_{3}$ & $\mathrm{CCOP}(\mathrm{OCC}) \mathrm{OCC}$ & $\begin{array}{l}\text { (-10(8-5-2)9-6-3/h4-6H2,1- } \\
3 \mathrm{H} 3 \\
\text { InChIKey=BDZBKCUKTQZU } \\
\text { TL-UHFFFAOYSA-N }\end{array}$ & AG 30 & $\begin{array}{l}\text { (MRV). } \\
\text { Show 3D Structure } \\
\text { NIST Chemistry } \\
\text { WebBook }\end{array}$ \\
\hline Precursors & CWC 3B10 & Dimethyl phosphite & 868-85-9 & $\underline{6327114}$ & $0-\left.\right|_{H} ^{P}-0$ & {$[\mathrm{H}] \mathrm{P}(=\mathrm{O})(\mathrm{OC}) \mathrm{OC}$} & $\begin{array}{l}\text { InChl=1S/C2H7O3P/c1-4- } \\
6(3) 5-2 / h 6 \mathrm{H}, 1-2 \mathrm{H} 3 \\
\text { InChIKey=YLFBFPXKTIQSS } \\
\text { Y-UHFFFAOYSA-N }\end{array}$ & AG 6 & $\begin{array}{l}\text { Download 2D Structure } \\
\text { (DF). } \\
\text { Download 2D Structure } \\
\text { (MRV). } \\
\text { Show 3D Structure } \\
\text { NIST Chemistry. } \\
\text { WebBook }\end{array}$ \\
\hline Precursors & CWC 3B11 & Diethyl phosphite & $762-04-9$ & $\underline{6327654}$ & 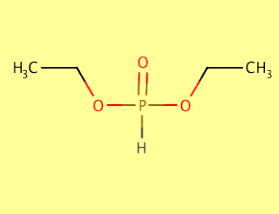 & {$[\mathrm{H}] \mathrm{P}(=\mathrm{O})(\mathrm{OCC}) \mathrm{OCC}$} & $\begin{array}{l}\text { InChl=1S/C4H11O3P/c1-3- } \\
6-8(5) 7-4-2 / \mathrm{h} 8 \mathrm{H}, 3-4 \mathrm{H} 2,1- \\
2 \mathrm{H} 3 \\
\text { InChIKey=LXCYSACZTOKN } \\
\text { NS-UHFFFAOYSA-N }\end{array}$ & AG 19 & $\begin{array}{l}\text { Download 2D Structure } \\
\text { (SDF). } \\
\text { Download 2D Structure } \\
\text { (MRV). } \\
\text { Show 3D Structure } \\
\text { NIST Chemistry. } \\
\text { WebBook }\end{array}$ \\
\hline Precursors & CWC 3B12 & Sulfur monochloride & $10025-67-9$ & $\underline{24807}$ & $\mathrm{Cl}$ & $\mathrm{ClSSCl}$ & $\begin{array}{l}\text { InChl=1S/CI2S2/c1-3-4-2 } \\
\text { InChIKey=PXJJSXABGXMU } \\
\text { SU-UHFFFAOYSA-N }\end{array}$ & AG 51 & $\begin{array}{l}\text { Download 2D Structure } \\
\text { (SDF). } \\
\text { Download 2D Structure } \\
\text { (MRV). } \\
\text { Show 3D Structure } \\
\text { NIST Chemistry. } \\
\text { WebBook }\end{array}$ \\
\hline Precursors & CWC 3B13 & Sulfur dichloride & $10545-99-0$ & $\underline{25353}$ & & $\mathrm{CISCl}$ & $\begin{array}{l}\text { InChl=1S/Cl2S/c1-3-2 } \\
\text { InChIKey=FWMUJAIKEJWS } \\
\text { SY-UHFFFAOYSA-N }\end{array}$ & AG 52 & $\begin{array}{l}\text { Download 2D Structure } \\
\text { (SDF). } \\
\text { Download 2D Structure } \\
\text { (MRV). } \\
\text { Show 3D Structure } \\
\text { NIST Chemistry. } \\
\text { WebBook }\end{array}$ \\
\hline Precursors & CWC 3B14 & Thionyl chloride & 7719-09-7 & $\underline{24386}$ & & $\mathrm{Cl}[\mathrm{S}](\mathrm{Cl})=\mathrm{O}$ & $\begin{array}{l}\text { InChl=1S/CI2OS/c1-4(2)3 } \\
\text { InChIKey=FYSNRJHAOHDI } \\
\text { LO-UHFFFAOYSA-N }\end{array}$ & AG 9 & $\begin{array}{l}\text { Download 2D Structure } \\
\text { (SDF). } \\
\text { Download 2D Structure } \\
\text { (MRV). } \\
\text { Show 3D Structure } \\
\text { NIST Chemistry } \\
\text { WebBook }\end{array}$ \\
\hline Precursors & CWC 3B15 & Ethyldiethanolamine & $139-87-7$ & $\underline{8769}$ & & 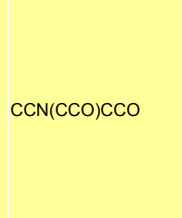 & $\begin{array}{l}\text { InChl=1S/C6H15NO2/c1-2- } \\
\text { 7(3-5-8)4-6-9/h8-9H,2- } \\
\text { 6H2,1H3 } \\
\text { InChIKey=AKNUHUCEWAL } \\
\text { COI-UHFFFAOYSA-N }\end{array}$ & AG 59 & $\begin{array}{l}\text { Download 2D Structure } \\
\text { ( } \text { (SDF) } \\
\text { Download 2D Structure } \\
\text { (MRV) } \\
\text { Show 3D Structure } \\
\text { NIST Chemistry. } \\
\text { WebBook }\end{array}$ \\
\hline Precursors & CWC 3B16 & Methyldiethanolamine & $105-59-9$ & $\underline{7767}$ & & $\mathrm{CN}(\mathrm{CCO}) \mathrm{CCO}$ & $\begin{array}{l}\text { InChl=1S/C5H13NO2/c1- } \\
6(2-4-7) 3-5-8 / h 7-8 \mathrm{H}, 2- \\
5 \mathrm{H} 2,1 \mathrm{H} 3 \\
\text { InChIKey=CRVGTESFCCX } \\
\text { CTH-UHFFFAOYSA-N }\end{array}$ & - & $\begin{array}{l}\text { Download 2D Structure } \\
\text { (SDF). } \\
\text { Download 2D Structure } \\
\text { (MRV). } \\
\text { Show 3D Structure } \\
\text { NIST Chemistry } \\
\text { WebBook }\end{array}$ \\
\hline Precursors & CWC 3B17 & Triethanolamine & $102-71-6$ & $\underline{7618}$ & & $\mathrm{OCCN}(\mathrm{CCO}) \mathrm{CCO}$ & $\begin{array}{l}\text { InChl=1S/C6H15NO3/C8-4- } \\
1-7(2-5-9) 3-6-10 / h 8-10 \mathrm{H}, 1- \\
6 \mathrm{H} 2 \\
\text { InChIKey=GSEJCLTVZPLZK } \\
\text { Y-UHFFFAOYSA-N }\end{array}$ & AG 46 & $\begin{array}{l}\frac{\text { Download 2D Structure }}{\text { (SDF). }} \\
\text { Download 2D Structure } \\
\text { (MRV). } \\
\text { Show 3D Structure } \\
\text { NIST Chemistry. } \\
\text { WebBook }\end{array}$ \\
\hline
\end{tabular}


Table S2. Annotated Australia Group (AG) Chemical Weapons Precursors List - also available on the Costanzi Research Website at: https://costanziresearch.com/cw-nonproliferation/cwcontrol-lists/ag-chemicals/ 


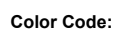

$\underline{12958}$

\begin{tabular}{|c|c|c|c|c|}
\hline Precursor & AG 3 & $\begin{array}{l}\text { Dimethyl } \\
\text { methylphosphonate }\end{array}$ & $756-79-6$ & $\underline{12958}$ \\
\hline Precursor & AG 4 & $\begin{array}{l}\text { Methylphosphonyl } \\
\text { difluoride (DF) }\end{array}$ & $676-99-3$ & $\underline{69610}$ \\
\hline Precursor & AG 5 & $\begin{array}{l}\text { Methylphosphonyl } \\
\text { dichloride (DC) }\end{array}$ & $676-97-1$ & $\underline{12671}$ \\
\hline Precursor & AG 6 & Dimethyl phosphite & $868-85-9$ & 63 \\
\hline
\end{tabular}

Precursor<smiles>CO[PH](=O)OC</smiles>

$[H] P(=O)(O C) O C$<smiles>CP(=O)(F)F</smiles>

$\mathrm{C}[\mathrm{P}](\mathrm{Cl})(\mathrm{Cl})=\mathrm{O}$<smiles>CP(=O)(Cl)Cl</smiles>

$\mathrm{Chl}=1 \mathrm{~S} / \mathrm{CH} 3 \mathrm{Cl} 2 \mathrm{OP} / \mathrm{c} 1$ $5(2,3) 4 / \mathrm{h} 1 \mathrm{H} 3$

InChl=1S/Cl3OP/c1-5(2,3)4 InChIKey=XHXFXVLFKHQF CWC 3B5
AL-UHFFFAOYSA-N

InChl=1S/C3H9O3P/c1-57(3,4)6-2/h1-3H3 InChIKey=VONWDA DY-UHFFFAOYSA-N

\section{InChl=1S/CH3F2OP/c1- $5(2,3) 4 / \mathrm{h} 1 \mathrm{H} 3$}

$\mathrm{C}[\mathrm{P}](\mathrm{F})(\mathrm{F})=\mathrm{O}$

ChIKey=PQIOS RI-UHFFFAOYSA-N

CWC 1B9 Example 1

WA ML7 c.1

$\mathrm{Chl}=1 \mathrm{~S} / \mathrm{C} 2 \mathrm{H} 703 \mathrm{P} / \mathrm{c} 1-4$ $6(3) 5-2 / h 6 H, 1-2 \mathrm{H} 3$

InChIKey=YLFBFPXKTIQSS Y-UHFFFAOYSA-N

CWC 3B10

InChl=1S/Cl3P/c1-4(2)3<smiles>ClP(Cl)Cl</smiles><smiles>COP(OC)OC</smiles>

$\mathrm{CIP}(\mathrm{Cl}) \mathrm{Cl}$

\section{InChIKey=FAIAAWCVCHQX CWC 3B6 DN-UHFFFAOYSA-N}

COP(OC)OC
AG $8 \quad$ Trimethyl phosphite $\quad 121-45-9 \quad \underline{8472}$

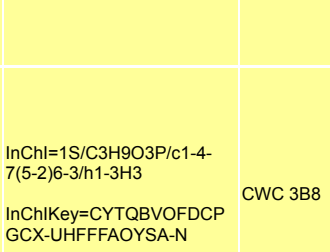

mpiled by the National
tra.

Download 2D Structure (SDF)

Download 2D Structure (MRV).

Show 3D Structure

NIST Chemistry

WebBook

Download 2D Structure (SDF).

Download 2D Structure (MRV).

Show 3D Structure

NIST Chemistry

WebBook

Download 2D Structure ( $\underline{\text { SDF). }}$

Download 2D Structure (MRV).

Show 3D Structure

NIST Chemistry

WebBook

Download 2D Structure (SDF)

Download 2D Structure (MRV)

Show 3D Structure

NIST Chemistry

WebBook

Download 2D Structure (SDF).

Download 2D Structure (MRV).

Show 3D Structure

NIST Chemistry WebBook

Download 2D Structure (SDF).

Download 2D Structure (MRV)

Show 3D Structure

NIST Chemistry.

WebBook

Download 2D Structure (SDF).

Download 2D Structure (MRV).

Show 3D Structure

NIST Chemistry WebBook

Download 2D Structure (SDF).

Download 2D Structure (MRV). Show 3D Structure NIST Chemistry. WebBook 


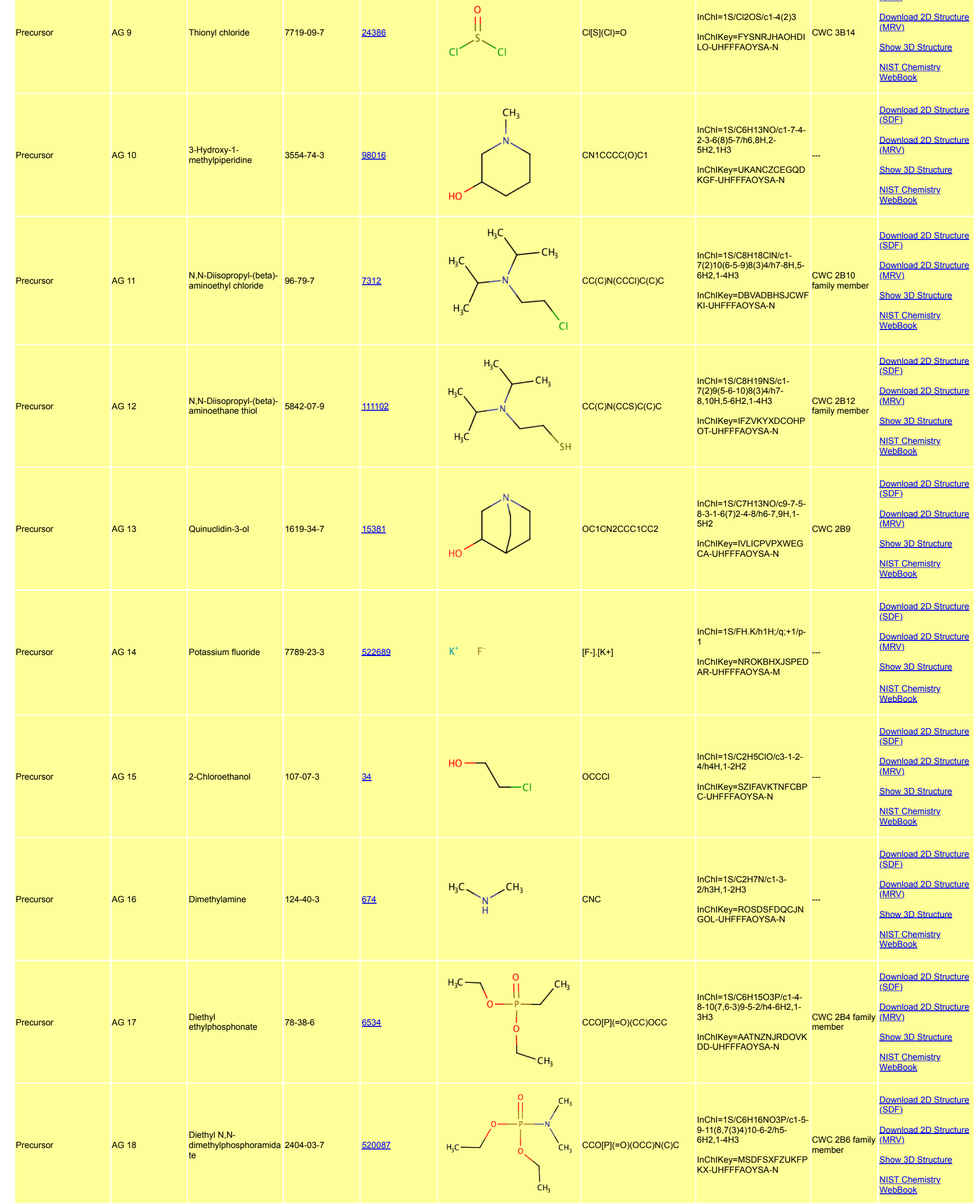




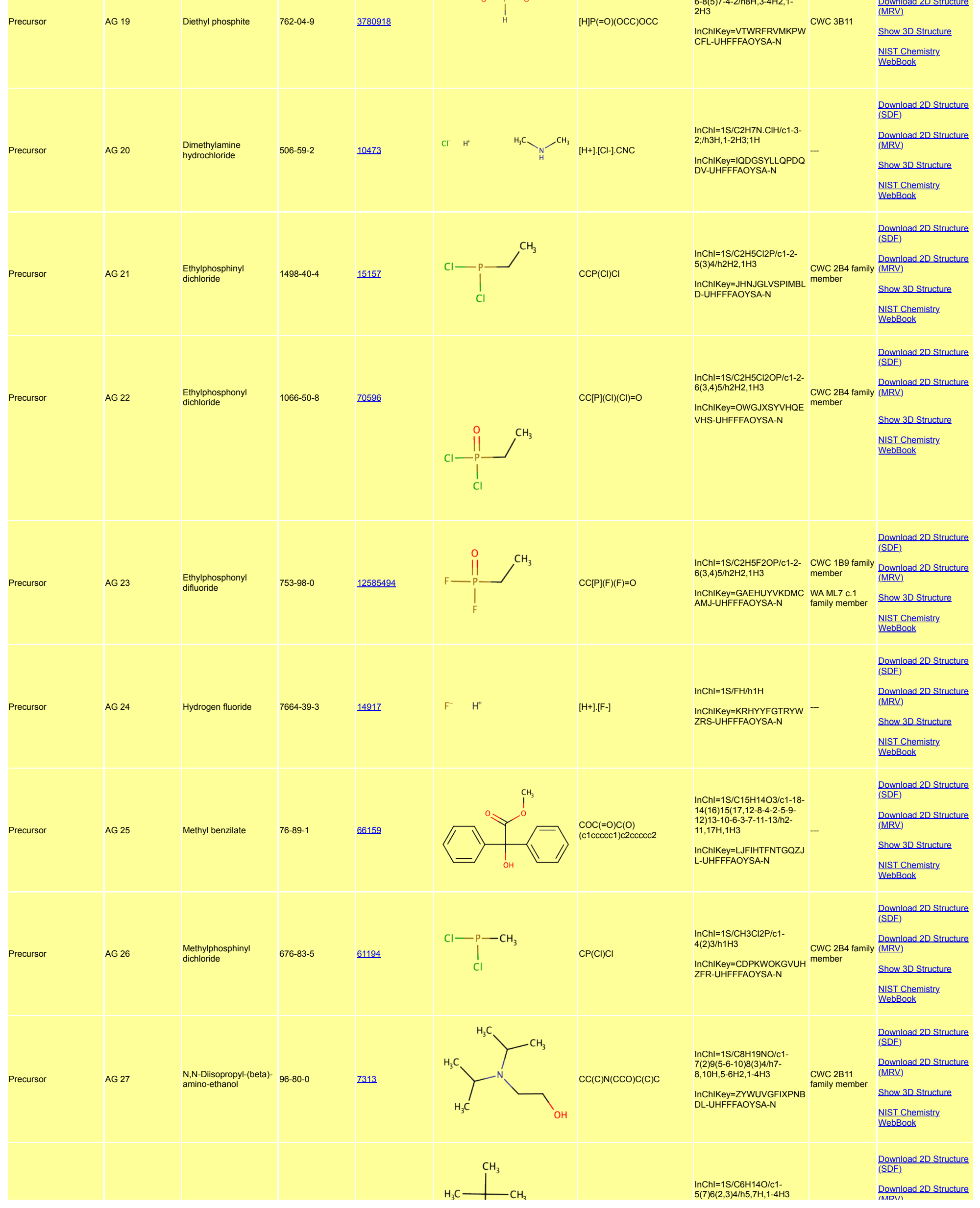




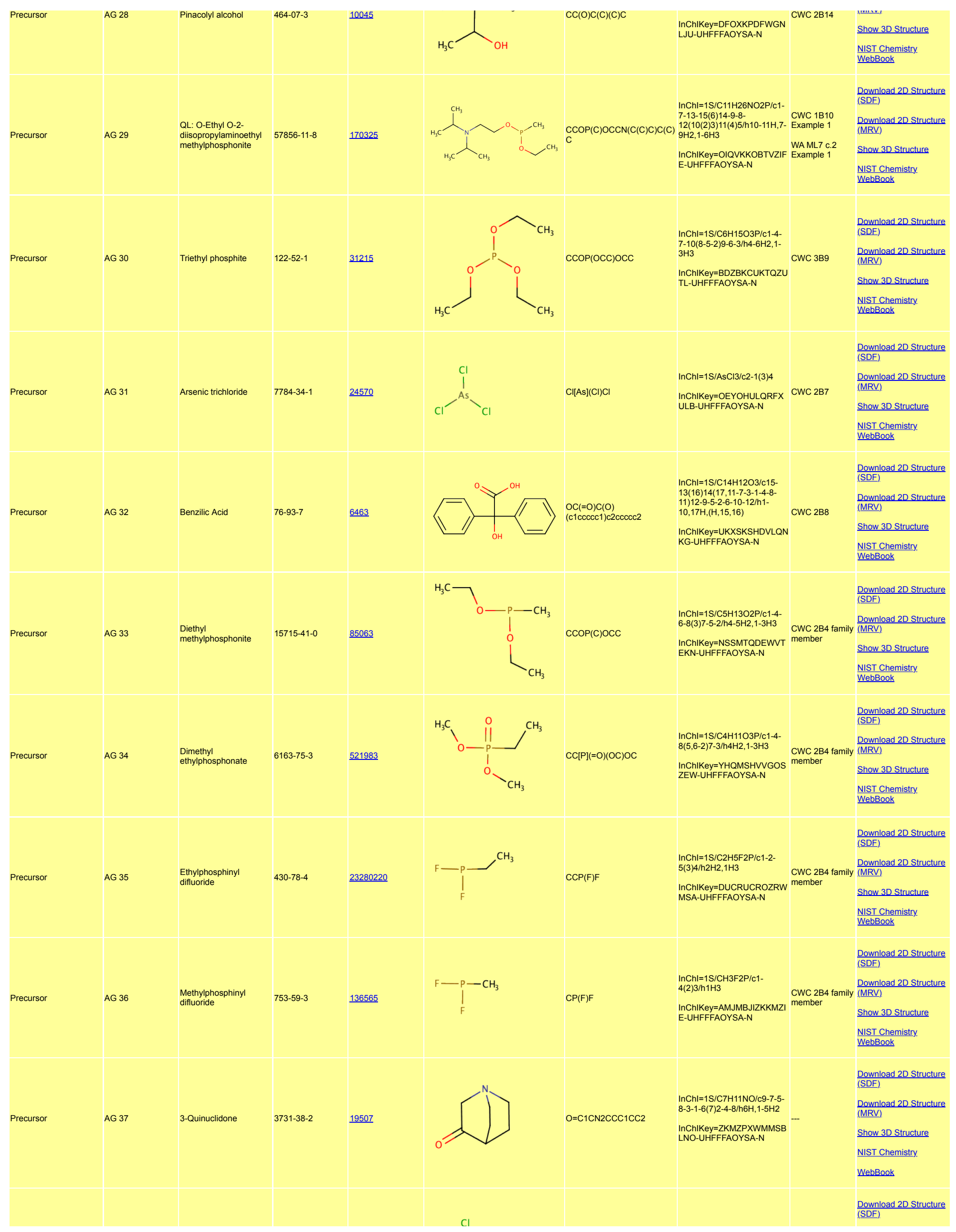




\begin{tabular}{|c|c|c|c|c|c|c|c|c|c|c|}
\hline Precursor & AG 38 & $\begin{array}{l}\text { Phosphorus } \\
\text { pentachloride }\end{array}$ & 10026-13-8 & $\underline{24819}$ & $\begin{array}{cl}C I & C l\end{array}$ & & $\mathrm{Cl}[\mathrm{P}](\mathrm{Cl})(\mathrm{Cl})(\mathrm{Cl}) \mathrm{Cl}$ & $\begin{array}{l}\text { InChl=1S/CISP/C1-6(2,3,4)5 } \\
\text { InChIKey=UHZYTMXLRWX } \\
\text { GPK-UHFFFAOYSA-N }\end{array}$ & CWC 3B7 & $\begin{array}{l}\text { Download 2D Structure } \\
\text { (MRV). } \\
\text { Show 3D Structure } \\
\text { NIST Chemistry. } \\
\text { WebBook }\end{array}$ \\
\hline Precursor & AG 39 & Pinacolone & $75-97-8$ & $\underline{6416}$ & $\mathrm{H}_{3} \mathrm{C}-\underbrace{\mathrm{CH}_{3} \mathrm{C}}_{\mathrm{O}} \mathrm{CH}_{3}$ & & $\mathrm{CC}(=0) \mathrm{C}(\mathrm{C})(\mathrm{C}) \mathrm{C}$ & $\begin{array}{l}\text { InChl=1S/C6H12O/c1- } \\
5(7) 6(2,3) 4 / \mathrm{h} 1-4 \mathrm{H} 3 \\
\text { InChIKey=PJGSXYOJTGTZ } \\
\text { AV-UHFFFAOYSA-N }\end{array}$ & - & $\begin{array}{l}\text { Download 2D Structure } \\
\text { (SDF). } \\
\text { Download 2D Structure } \\
\text { (MRV). } \\
\text { Show 3D Structure } \\
\text { NIST Chemistry. } \\
\text { WebBook }\end{array}$ \\
\hline Precursor & AG 40 & Potassium cyanide & $151-50-8$ & $\underline{9032}$ & $\mathrm{~K}^{+} \quad \mathrm{C} \equiv \mathrm{N}$ & & {$[\mathrm{K}+] \cdot[\mathrm{C}-] \# \mathrm{~N}$} & $\begin{array}{l}\text { InChl=1S/CN.K/c1-2;/q-1;+1 } \\
\text { InChIKey=NNFCIKHAZHQZ } \\
\text { JG-UHFFFAOYSA-N }\end{array}$ & & $\begin{array}{l}\frac{\text { Download 2D Structure }}{\text { (SDF). }} \\
\text { Download 2D Structure } \\
\text { (MRV). } \\
\text { Show 3D Structure } \\
\text { NIST Chemistry. } \\
\text { WebBook }\end{array}$ \\
\hline Precursor & AG 41 & Potassium bifluoride & 7789-29-9 & 11829350 & $\mathrm{~F}^{-} \quad \mathrm{H}^{+}$ & $\mathrm{K}^{+} \quad \mathrm{F}^{-}$ & {$[\mathrm{H}+] \cdot[\mathrm{F}-] \cdot[\mathrm{F}-] \cdot[\mathrm{K}+]$} & $\begin{array}{l}\text { InChl=1S/2FH.K/h2*1H;/q;:+ } \\
\text { 1/p-1 } \\
\text { InChIKey=VBKNTGMWIPU } \\
\text { CRF-UHFFFAOYSA-M }\end{array}$ & - & $\begin{array}{l}\text { Download 2D Structure } \\
\text { (SDF). } \\
\text { Download 2D Structure } \\
\text { (MRV). } \\
\text { Show 3D Structure } \\
\text { NIST Chemistry. } \\
\text { WebBook }\end{array}$ \\
\hline Precursor & AG 42 & Ammonium bifluoride & $1341-49-7$ & $\underline{14935}$ & $\mathrm{~F}^{-} \quad \mathrm{H}^{*}$ & $\mathrm{~F}^{-} \quad \mathrm{NH}_{4}^{+}$ & {$[\mathrm{H}+] \cdot[\mathrm{NH} 4+] .[\mathrm{F}-] .[\mathrm{F}-]$} & $\begin{array}{l}\text { InChl=1S/2FH.H3N/h2* } 1 \mathrm{H} ; 1 \\
\mathrm{H} 3 \\
\text { InChlKey=KVBCYCWRDBD } \\
\text { GBG-UHFFFAOYSA-N }\end{array}$ & - & $\begin{array}{l}\text { Download 2D Structure } \\
\text { (SDF). } \\
\text { Download 2D Structure } \\
\text { (MRV). } \\
\text { Show 3D Structure } \\
\text { NIST Chemistry. } \\
\text { WebBook }\end{array}$ \\
\hline Precursor & AG 43 & Sodium bifluoride & $1333-83-1$ & $\underline{219061}$ & $\mathrm{~F}^{-} \quad \mathrm{H}^{+}$ & $\mathrm{Na}^{+} \quad \mathrm{F}^{-}$ & {$[\mathrm{H}+] \cdot[\mathrm{F}-] \cdot[\mathrm{F}-] \cdot[\mathrm{Na}+]$} & $\begin{array}{l}\text { InChl=1S/2FH.Na/h2*1H;/q; } \\
+1 / p-1 \\
\text { InChIKey=BFXAWOHHDUIA } \\
\text { LU-UHFFFAOYSA-M }\end{array}$ & -- & $\begin{array}{l}\text { Download 2D Structure } \\
\text { (SDF). } \\
\text { Download 2D Structure } \\
\text { (MRV). } \\
\text { Show 3D Structure } \\
\text { NIST Chemistry. } \\
\text { WebBook }\end{array}$ \\
\hline Precursor & AG 44 & Sodium fluoride & 7681-49-4 & $\underline{5235}$ & $\mathrm{Na}^{+} \quad \mathrm{F}^{-}$ & & {$[\mathrm{F}] \cdot[\mathrm{Na}+]$} & $\begin{array}{l}\text { InChl=1S/FH.Na/h1H:/q;+1/p } \\
-1 \\
\text { InChIKey=PUZPDOWCWNU } \\
\text { UKD-UHFFFOYSA-M }\end{array}$ & -- & $\begin{array}{l}\text { Download 2D Structure } \\
\text { (SDF). } \\
\text { Download 2D Structure } \\
\text { (MRV). } \\
\text { Show 3D Structure } \\
\text { NIST Chemistry. } \\
\text { WebBook }\end{array}$ \\
\hline Precursor & AG 45 & Sodium cyanide & 143-33-9 & 8929 & $\mathrm{Na}^{+} \quad \mathrm{C} \equiv \mathrm{N}$ & & {$[\mathrm{Na}+] \cdot[\mathrm{C}-] \# \mathrm{~N}$} & $\begin{array}{l}\text { InChl=1S/CN.Na/c1-2;/q- } \\
1 ;+1 \\
\text { InChIKey=MNWBNISUBARL } \\
\text { IT-UHFFFAOYSA-N }\end{array}$ & -- & $\begin{array}{l}\text { Download 2D Structure } \\
\text { (SDF). } \\
\text { Download 2D Structure } \\
\text { (MRV). } \\
\text { Show 3D Structure } \\
\text { NIST Chemistry. } \\
\text { WebBook }\end{array}$ \\
\hline Precursor & AG 46 & Triethanolamine & $102-71-6$ & $\underline{7618}$ & & & $\mathrm{OCCN}(\mathrm{CCO}) \mathrm{CCO}$ & $\begin{array}{l}\text { InChl=1S/C6H15NO3/C8-4- } \\
1-7(2-5-9) 3-6-10 / h 8-10 \mathrm{H}, 1- \\
6 \mathrm{H} 2 \\
\text { InChIKey=GSEJCLTVZPLZK } \\
\text { Y-UHFFFAOYSA-N }\end{array}$ & CWC 3B17 & $\begin{array}{l}\frac{\text { Download 2D Structure }}{\text { (SDF). }} \\
\text { Download 2D Structure } \\
\text { (MRV). } \\
\text { Show 3D Structure } \\
\text { NIST Chemistry. } \\
\text { WebBook }\end{array}$ \\
\hline Precursor & AG 47 & $\begin{array}{l}\text { Phosphorus } \\
\text { pentasulphide }\end{array}$ & 1314-80-3 & 14817 & $s=$ & & $\begin{array}{l}\mathrm{S}=[\mathrm{P}] 12 \mathrm{~S}[\mathrm{P}] 3(=\mathrm{S}) \mathrm{S}[\mathrm{P}]=\mathrm{S}) \\
(\mathrm{S} 1) \mathrm{S}[\mathrm{P}](=\mathrm{S})(\mathrm{S} 2) \mathrm{S} 3\end{array}$ & $\begin{array}{l}\text { InChl=1S/P4S10/c5-1-9- } \\
2(6) 12-3(7,10-1) 14-4(8,11- \\
\text { 1)13-2 } \\
\text { InChIKey=CYQAYERJWZKKY } \\
\text { ML-UHFFFAOYSA-N }\end{array}$ & 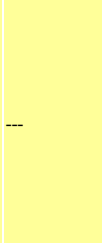 & $\begin{array}{l}\frac{\text { Downnload 2D Structure }}{\text { (SDF). }} \\
\text { Download 2D Structure } \\
\text { (MRV). } \\
\text { Show 3D Structure } \\
\text { NIST Chemistry } \\
\text { WebBook }\end{array}$ \\
\hline
\end{tabular}




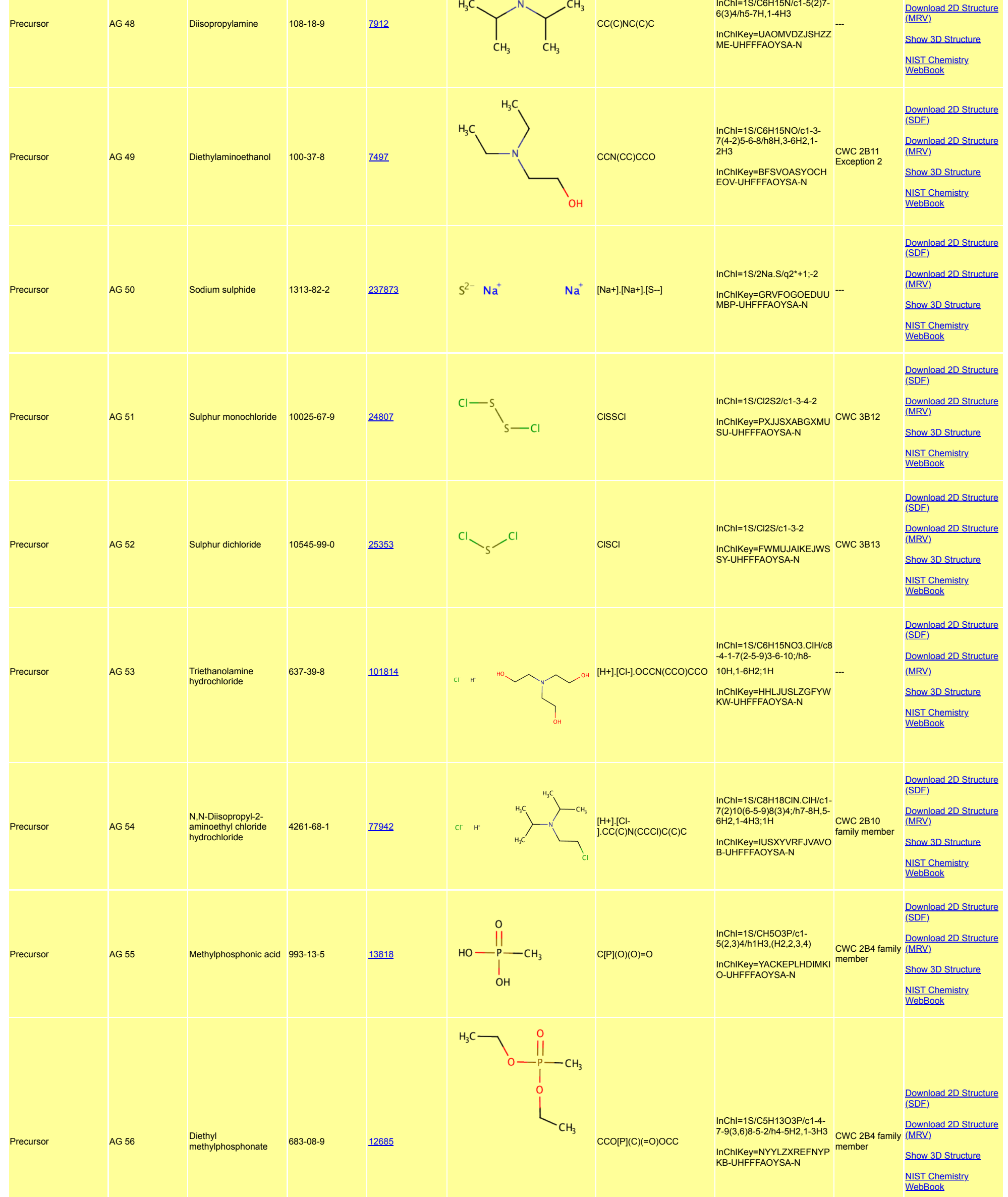




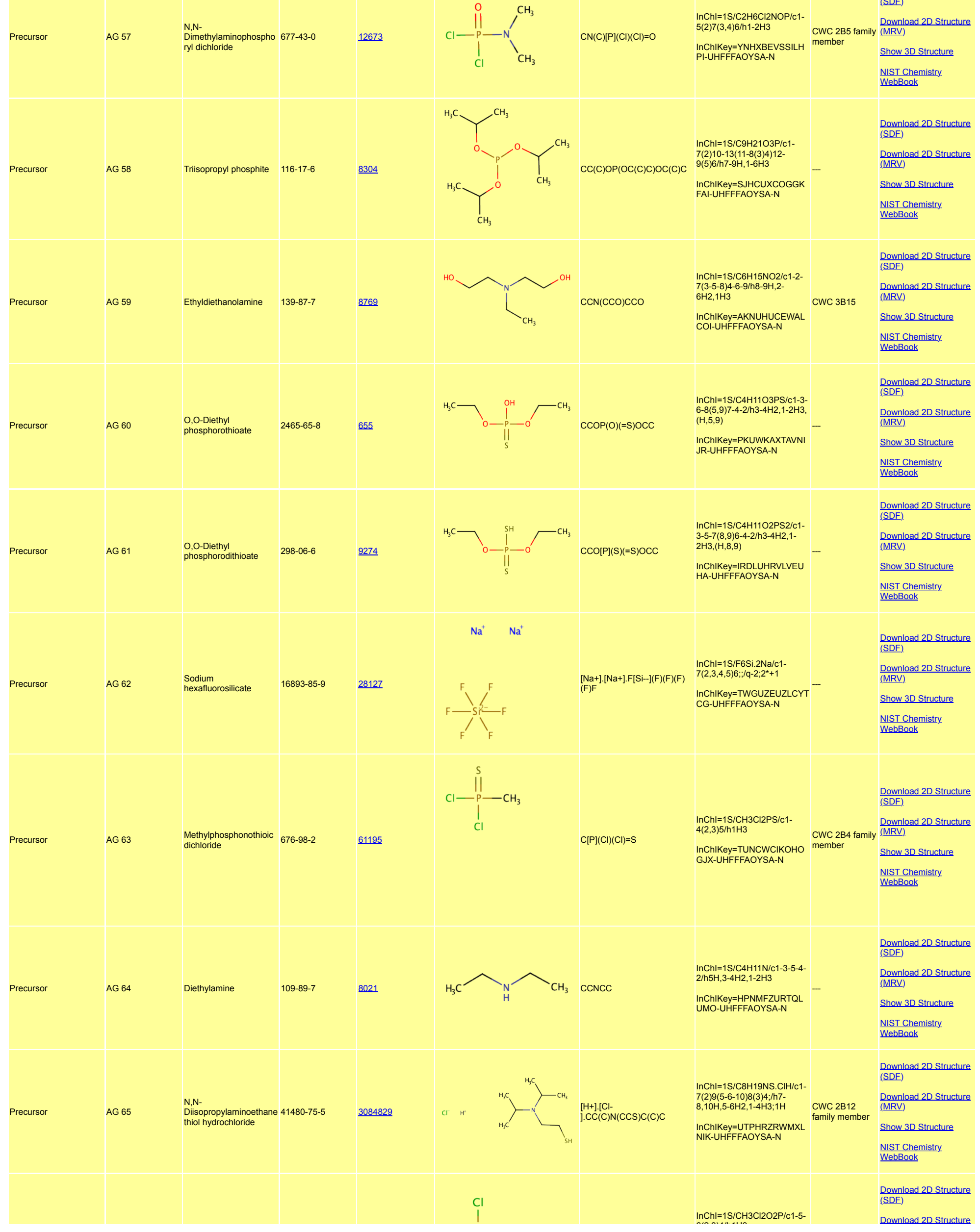




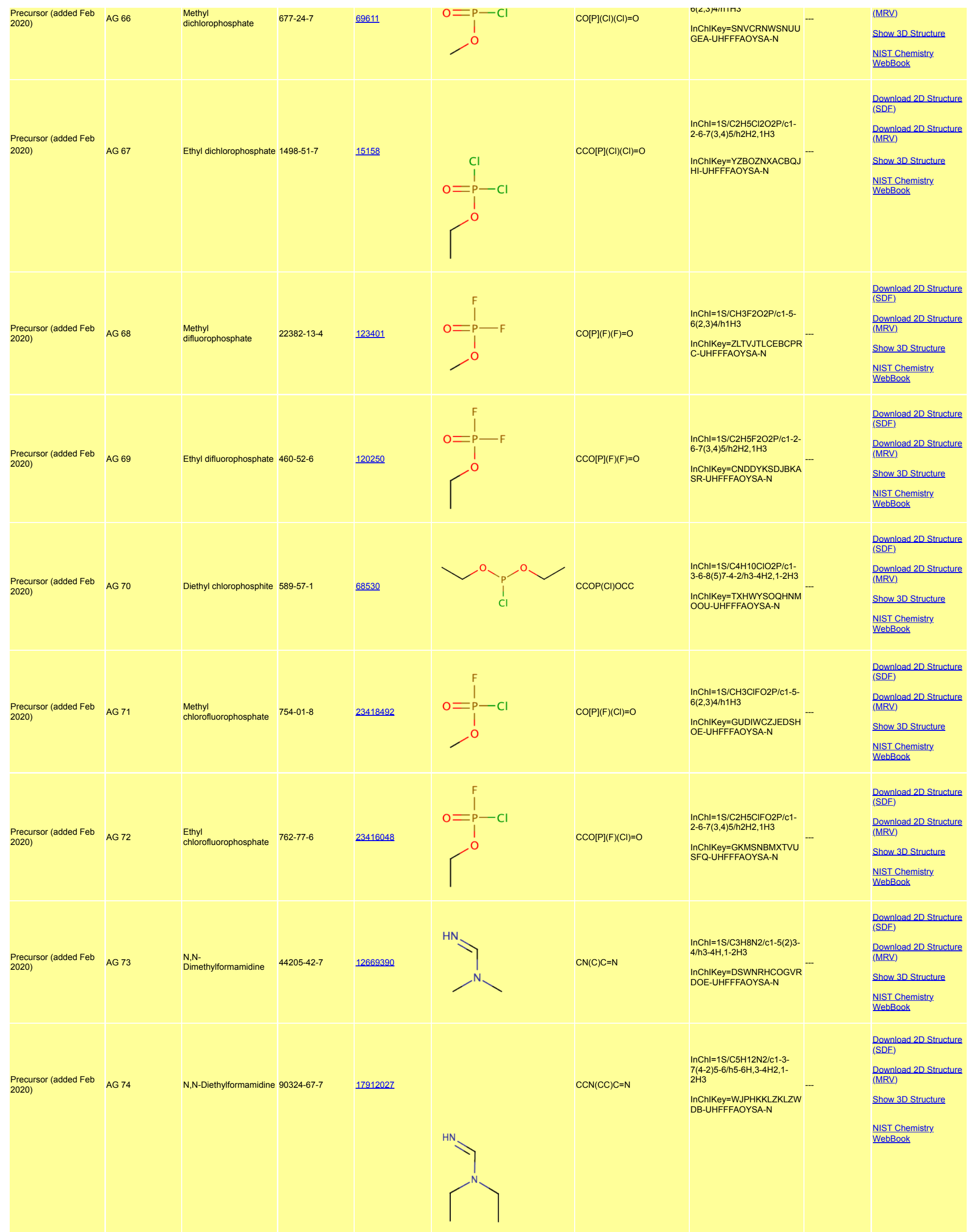




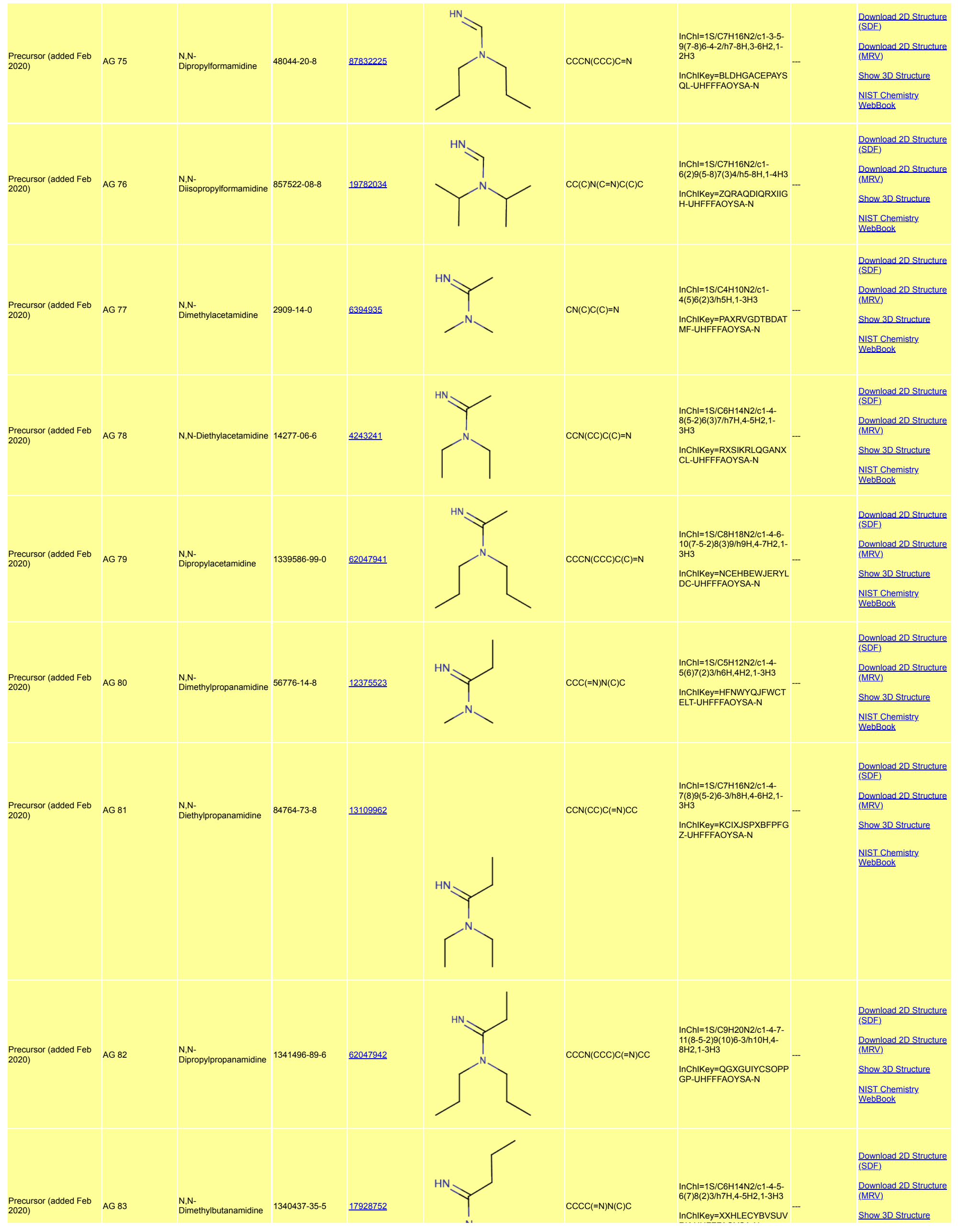




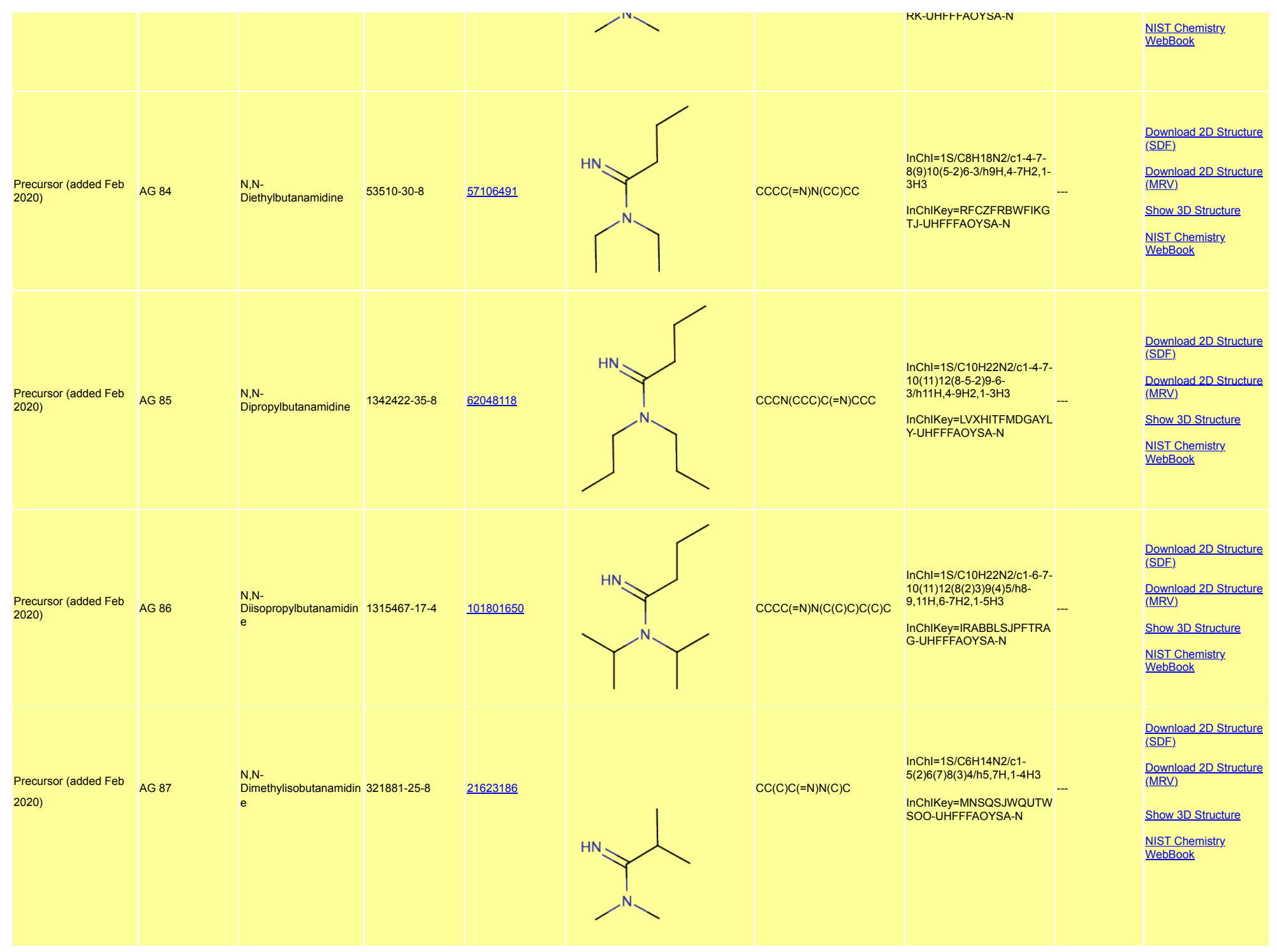


Table S3. Annotated Wassenaar Arrangement (WA) Munitions List 7 (ML7) - also available on the Costanzi Research Website at: https://costanziresearch.com/cw-nonproliferation/cw-controllists/wassenaar-ml7/ 
Wassenaar Arrangement's Munitions List 7 (ML7) Chemicals

Curated and Structurally Annotated by Costanzi Research - www.costanziresearch.com (Last Update: September 03, 2020)

The Table is available for download in the following formats:

SDF, CSV, Microsoft Excel

Isclaimer. The information in this table is provided for research and general information purposes only. The authors do not guarantee the accuracy of the information and do not take any that may occur due to inaccuracies or omissions.

Color Code:

\section{Individual Chemicals}

Mixtures of Chemicals

\section{Proteins}

Families of Chemicals

Exceptions: Compounds Belonging to a Family of Chemicals but Excluded from the CWC Schedules

Notes.

1. For entry ML7 b.4.b (Agent Orange), there is a discrepancy between the name, which indicate the free acid form, and the CAS number, which indicates the butyl ester form.

. For entries lacking a CAS registry number, a sequential registry number assinged by Costanz Research (CR number) is provided instead.

3. For proteins, the UNIPROT ID is provided instead.

4. Overlaps among CWC schedules, Australia Group chemicals, and Wassenaar ML7 chemicals

5. $2 \mathrm{D}$ structures curated by Costanzi Research are provided as SDF and MRV files (MRV file

6. 3D strucures from PubChem are shown with NCBI's iCn3D.

Dhe Nation Institute of Standards and Technology, including, inter alia, analytical spectra.

\begin{tabular}{|c|c|c|c|c|c|c|c|c|c|}
\hline Category & $\begin{array}{c}\text { Wassenaar Entry } \\
\text { Number }^{1}\end{array}$ & $\begin{array}{c}\text { Wassenaar Entry } \\
\text { Name }\end{array}$ & $\begin{array}{l}\text { CAS Registry } \\
\text { Number }^{2}\end{array}$ & PubChem ID ${ }^{3}$ & $\begin{array}{c}\text { Structure } \\
\text { (click to enlarge) }\end{array}$ & SMILES & InChI & Overlaps $^{4}$ & Links $^{5-7}$ \\
\hline Nerve agents & WA ML7 b.1.a & $\begin{array}{l}\text { O-Alkyl (<=C10, incl. } \\
\text { cycloalkyl) alkyl (Me, Et, } \\
\text { n-Pr or i-Pr)- } \\
\text { phosphonofluoridates }\end{array}$ & CR0001 & --- & $\begin{array}{l}\mathrm{R} 1=\begin{array}{l}\mathrm{Me}, \mathrm{Et}, \mathrm{n}-\mathrm{Pr}, \\
\text { or } \mathrm{i}-\mathrm{Pr}\end{array} \\
\mathrm{R} 2=\begin{array}{l}\text { alkyl }(<=\mathrm{C} 10), \\
\text { incl. cycloalkyl }\end{array}\end{array}$ & --- & --- & CWC 1A1 & --- \\
\hline Nerve agents & $\begin{array}{l}\text { WA ML7 b. } 1 . a \\
\text { Example } 1\end{array}$ & $\begin{array}{l}\text { Sarin: O-Isopropyl } \\
\text { methylphosphonofluorid } \\
\text { ate }\end{array}$ & $107-44-8$ & $\underline{7871}$ & $=\overbrace{\mathrm{CH}_{3}}^{\mathrm{P}-\mathrm{CH}_{3}}$ & $\mathrm{CC}(\mathrm{C}) \mathrm{O}[\mathrm{P}](\mathrm{C})(\mathrm{F})=\mathrm{O}$ & $\begin{array}{l}\text { InChl=1S/C4H10FO2P/c1- } \\
4(2) 7-8(3,5) 6 / h 4 \mathrm{H}, 1-3 \mathrm{H} 3 \\
\text { InChIKey=DYAHQFWOVKZ } \\
\text { OOW-UHFFFAOYSA-N }\end{array}$ & $\begin{array}{l}\text { CWC } 1 \mathrm{~A} 1 \\
\text { Example } 1\end{array}$ & $\begin{array}{l}\text { Download 2D Structure } \\
\text { (SDF). } \\
\text { Download 2D Structure } \\
\text { (MRV). } \\
\text { Show 3D Structure } \\
\text { NIST Chemistry. } \\
\text { WebBook }\end{array}$ \\
\hline Nerve agents & $\begin{array}{l}\text { WA ML7 b.1.a } \\
\text { Example } 2\end{array}$ & $\begin{array}{l}\text { Soman: O-Pinacolyl } \\
\text { methylphosphonofluorid } \\
\text { ate }\end{array}$ & $96-64-0$ & $\underline{7305}$ & $\left.\right|_{\mathrm{CH}_{3}} \mathrm{CH}_{3}$ & $\mathrm{CC}(\mathrm{O}[\mathrm{P}](\mathrm{C})(\mathrm{F})=\mathrm{O}) \mathrm{C}(\mathrm{C})(\mathrm{C}) \mathrm{C}$ & $\begin{array}{l}\text { InChl=1S/C7H16FO2P/c1- } \\
6(7(2,3) 4) 10-11(5,8) 9 / h 6 \mathrm{H}, 1- \\
5 \mathrm{H3} \\
\text { InChIKey=GRXKLBBBQUKJ } \\
\text { JZ-UHFFFAOYSA-N }\end{array}$ & $\begin{array}{l}\text { CWC } 1 \mathrm{~A} 1 \\
\text { Example } 2\end{array}$ & $\begin{array}{l}\text { Download 2D Structure } \\
\text { (SDF). } \\
\text { Download 2D Structure } \\
\text { (MRV). } \\
\text { Show 3D Structure } \\
\text { NIST Chemistry } \\
\text { WebBook }\end{array}$ \\
\hline Nerve agents & WA ML7 b.1.b & $\begin{array}{l}\text { O-Alkyl (<=C10, incl. } \\
\text { cycloalkyl) N,N-dialkyl } \\
\text { (Me, Et, n-Pr or i-Pr) } \\
\text { phosphoramidocyanidat } \\
\text { es }\end{array}$ & CR0002 & --- & $\begin{array}{l}\mathrm{R} 1, \mathrm{R} 2=\begin{array}{l}\mathrm{Me}, \mathrm{Et}, \mathrm{n}-\mathrm{Pr}, \\
\text { or } \mathrm{i}-\mathrm{Pr}\end{array} \\
\mathrm{R} 3=\begin{array}{l}\text { alkyl }(<=\mathrm{C} 10), \\
\text { incl. cycloalkyl }\end{array}\end{array}$ & -- & --- & CWC 1 A2 & --- \\
\hline Nerve agents & $\begin{array}{l}\text { WA ML7 b.1.b } \\
\text { Example } 1\end{array}$ & $\begin{array}{l}\text { Tabun:O-Ethyl N,N- } \\
\text { dimethyl } \\
\text { phosphoramidocyanidat } \\
\text { e }\end{array}$ & 77-81-6 & $\underline{6500}$ & $\left.\mathrm{~N} \equiv\right|_{\mathrm{O}} ^{\mathrm{P}}=\mathrm{C}_{\mathrm{CH}_{3}}^{\mathrm{O}}$ & $\mathrm{CCO}[\mathrm{P}](=\mathrm{O})(\mathrm{CHN}) \mathrm{N}(\mathrm{C}) \mathrm{C}$ & $\begin{array}{l}\text { InChl=1S/C5H11N2O2P/c1- } \\
4-9-10(8,5-6) 7(2) 3 / h 4 H 2,1- \\
3 \mathrm{H} 3 \\
\text { InChIKey=PJVJTCIRVMBVI } \\
\text { A-UHFFFAOYSA-N }\end{array}$ & $\begin{array}{l}\text { CWC } 1 \mathrm{~A} 2 \\
\text { Example } 1\end{array}$ & $\begin{array}{l}\text { Download 2D Structure } \\
\text { (SDF). } \\
\text { Download 2D Structure } \\
\text { (MRV). } \\
\text { Show 3D Structure } \\
\text { NIST Chemistry. } \\
\text { WebBook }\end{array}$ \\
\hline Nerve agents & WA ML7 b.1.c & $\begin{array}{l}\text { O-Alkyl (H or }<=\mathrm{C} 10 \text {, } \\
\text { incl. cycloalkyl) S-2- } \\
\text { dialkyl (Me, Et, } n-P r \text { or i- } \\
\text { Pr)-aminoethyl alkyl } \\
\text { (Me, Et, } n \text {-Pr or i-Pr) } \\
\text { phosphonothiolates and } \\
\text { corresponding alkylated } \\
\text { or protonated salts }\end{array}$ & CR0003 & --- & 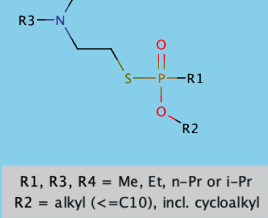 & --- & ... & CWC $1 A 3$ & -.. \\
\hline & & & & & $\mathrm{H}_{3} \mathrm{C}_{1}$ & & & & $\begin{array}{l}\text { Download 2D Structure } \\
\text { (SDF) }\end{array}$ \\
\hline
\end{tabular}




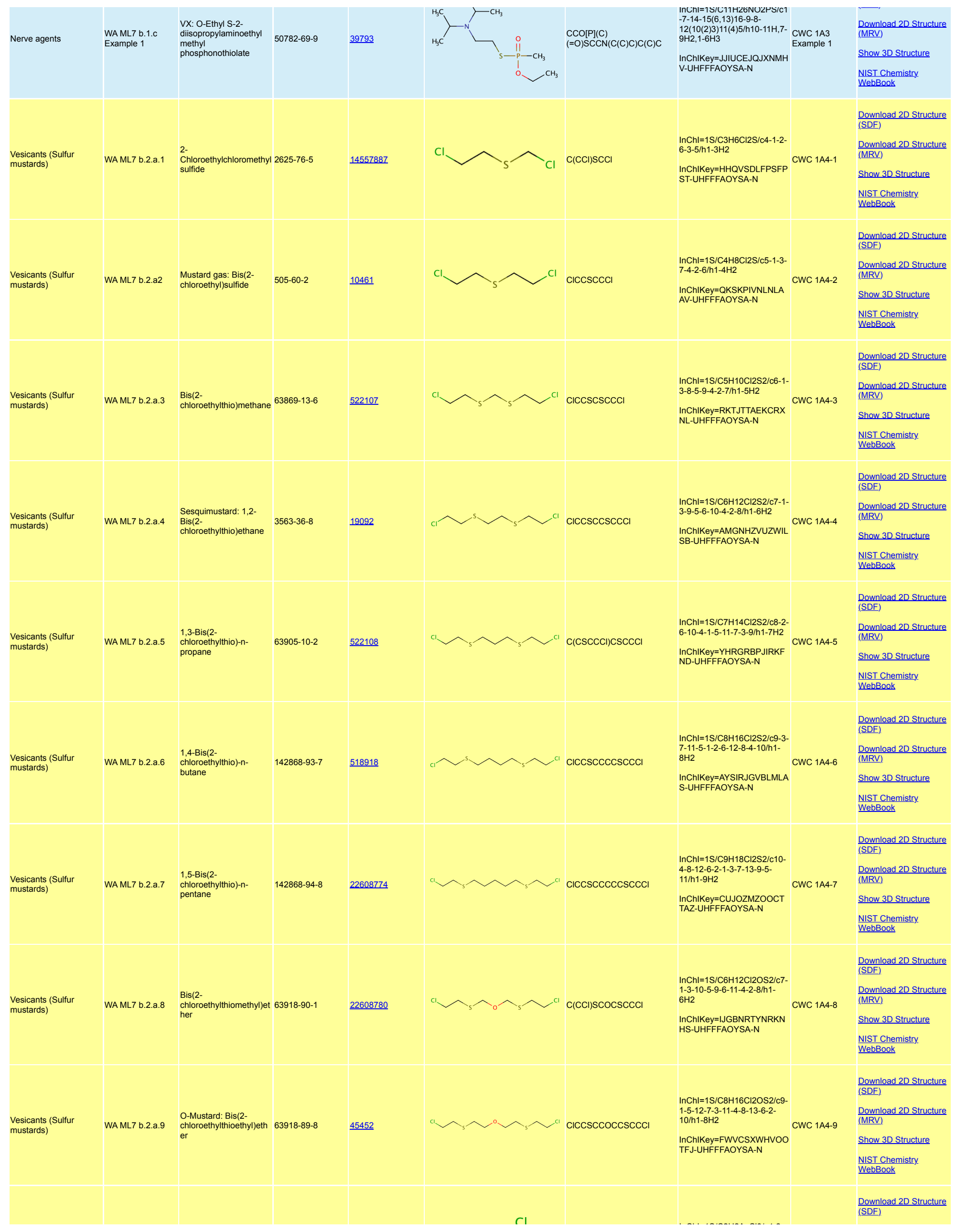




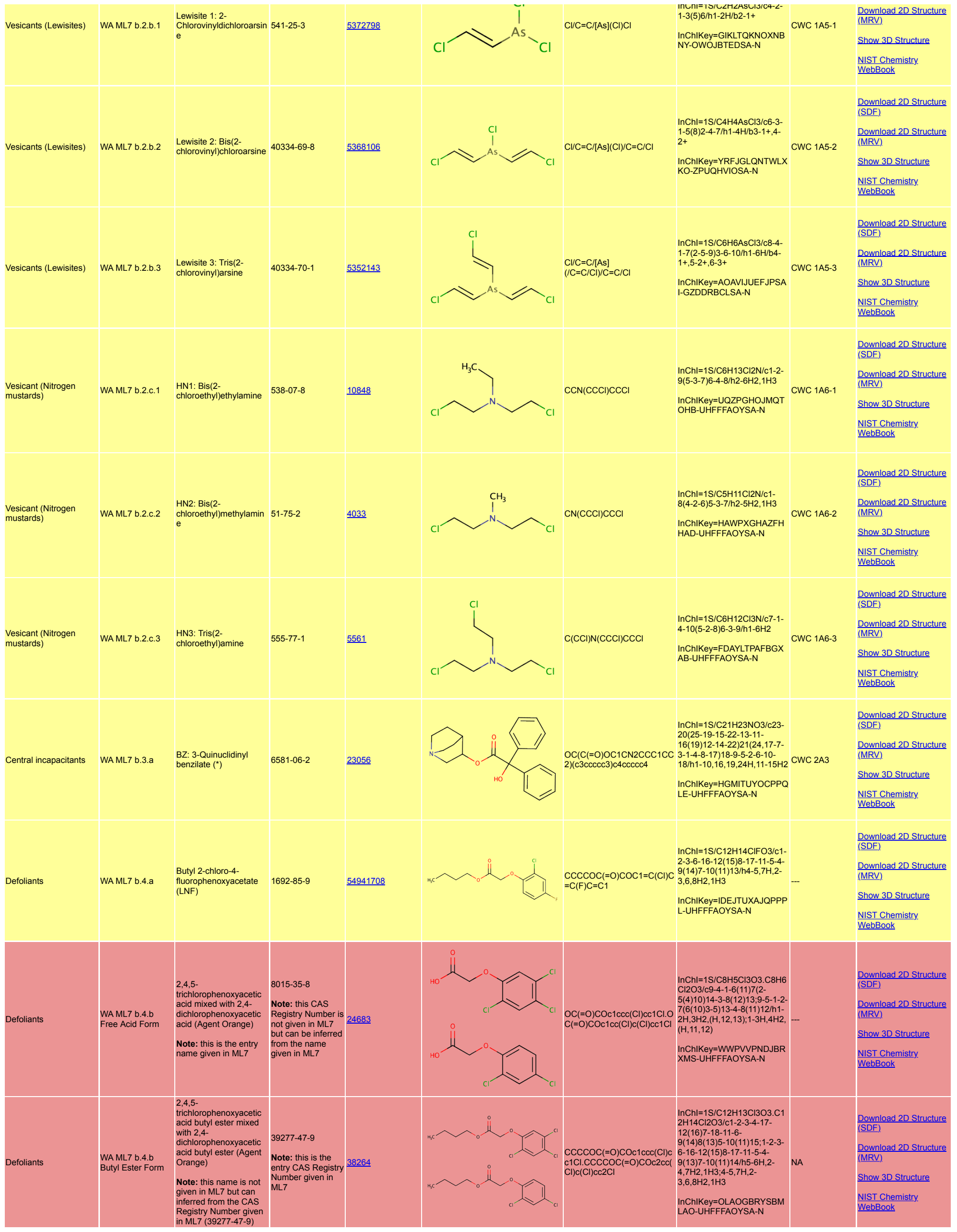



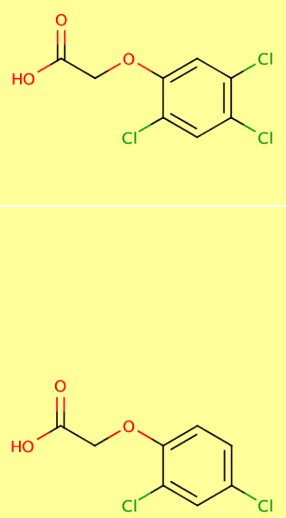

$\mathrm{OC}(=\mathrm{O}) \mathrm{CO} \operatorname{cocc}(\mathrm{Cl}) \mathrm{cct}$

1-2-7(6(10)3-5)13-4-

$8(11) 12 / \mathrm{h} 1-3 \mathrm{H}, 4 \mathrm{H} 2,(\mathrm{H}, 11,12)$

InChIKey=OVSKIKFHRZPJS S-UHFFFAOYSA-N
Download 2D Structure

Download 2D Structure

Show 3D Structure

NIST Chemistry WebBook

Download 2D Structure (SDF).

Download 2D Structure (MRV)

Show 3D Structure

NIST Chemistry WebBook

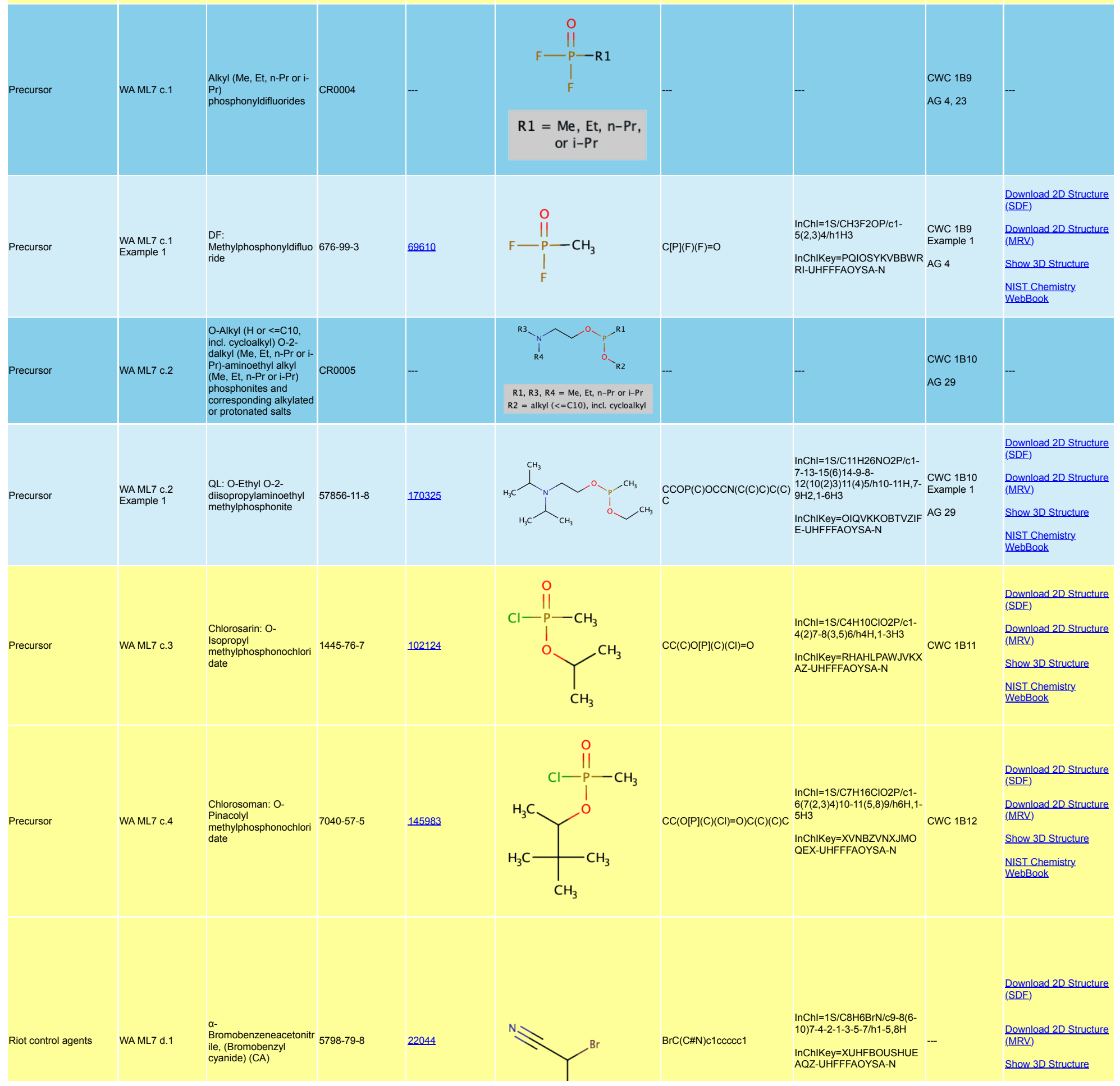




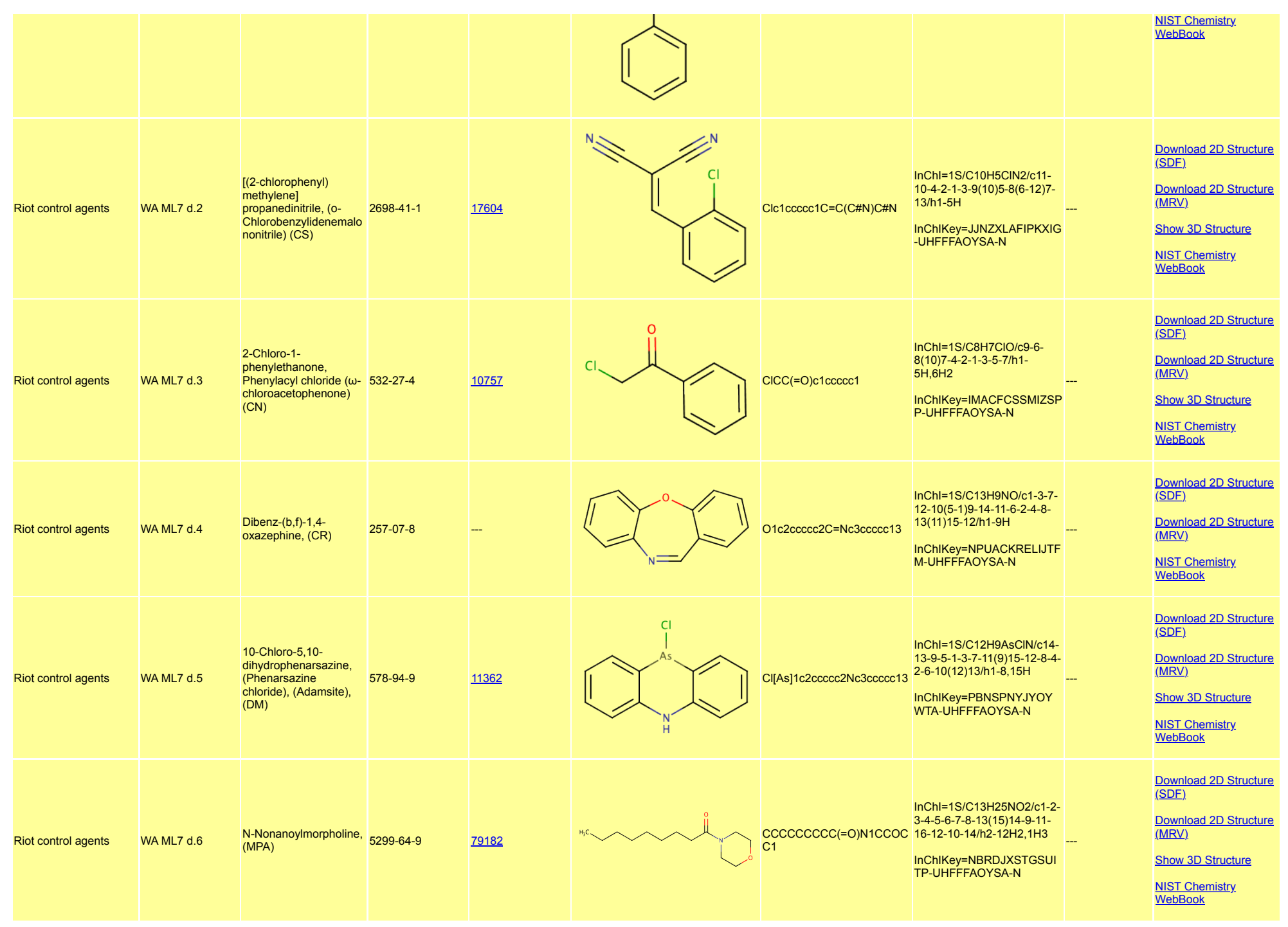


Table S4. Synoptic Table of CWC Schedules, AG Precursors List and WA ML7 - also available on the Costanzi Research Website at: https://costanziresearch.com/cw-nonproliferation/cwcontrol-lists/synoptic-table-of-cw-control-lists/ 


\section{Synoptic Table of CW-control lists}

Curated and Structurally Annotated by Costanzi Research - www.costanziresearch.com (Last Update: September 03, 2020)

The Table is available for download in the following formats:

CSV, Microsoft Excel

Color Code:

Individual Chemicals

Mixtures of Chemicals

Proteins

Families of Chemicals

Disclaimer. The information in this table is provided for research and general information purpose only. The authors do not guarantee the accuracy of the information and do not take any that may occur due to inaccuracies or omissions.

Notes.

1. When different names are given in different lists, the CWC name is provided first, followed by the name within parentheses.

2. For entries lacking a CAS registry number, a sequential registry number assinged by Costanz Research (CR number) is provided instead.

3. For proteins, the UNIPROT ID is provided instead. 4. Chemical Weapons Convention (CWC) entry num entered into force on June 7,2020.

5. Australia Group entry number. AG entries 66-87 were added on February 28, 2020. 6. Wassenaar Arrangement (WA) Munitions List 7 (ML7) entry number. For WA entry ML7 b.4.b (Agent Orange), there is a discrepancy between the name, which indicates the free acid form, and the CAS number, which indicates the butyl ester form.

\begin{tabular}{|c|c|c|c|c|c|c|}
\hline Category & Entry Name ${ }^{1}$ & $\begin{array}{l}\text { CAS Registry } \\
\text { Number }^{2}\end{array}$ & PubChem ID ${ }^{3}$ & CWC Entry Number ${ }^{4}$ & AG Entry Number ${ }^{5}$ & $\begin{array}{c}\text { Wassenaar ML7 Entry } \\
\text { Number }^{6}\end{array}$ \\
\hline Nerve agents & O-Alkyl ( $<=C 10$, incl. cycloalkyl) alkyl (Me, Et, n-Pr or i-Pr)-phosphonofluoridates & CR0001 & -- & CWC 1A1 & & WA ML7 b.1.a \\
\hline Nerve agents & Sarin: O-Isopropyl methylphosphonofluoridate & $107-44-8$ & $\underline{7871}$ & CWC 1 A1 Example 1 & & $\begin{array}{l}\text { WA ML7 b.1.a Example } \\
1\end{array}$ \\
\hline Nerve agents & Soman: O-Pinacolyl methylphosphonofluoridate & $96-64-0$ & $\underline{7305}$ & CWC 1 A1 Example 2 & & $\begin{array}{l}\text { WA ML7 b.1.a Example } \\
2\end{array}$ \\
\hline Nerve agents & O-Alkyl (<=C10, incl. cycloalkyl) N,N-dialkyl (Me, Et, n-Pr or i-Pr) phosphoramidocyanidates & CR0002 & -- & CWC 1 A2 & & WA ML7 b.1.b \\
\hline Nerve agents & Tabun:O-Ethyl N,N-dimethyl phosphoramidocyanidate & 77-81-6 & $\underline{6500}$ & CWC 1 A2 Example 1 & & $\begin{array}{l}\text { WA ML7 b.1.b Example } \\
1\end{array}$ \\
\hline Nerve agents & $\begin{array}{l}\text { O-Alkyl ( } \mathrm{H} \text { or }<=\mathrm{C} 10 \text {, incl. cycloalkyl) S-2-dialkyl (Me, Et, n-Pr or i-Pr)-aminoethyl alkyl (Me, Et, n- } \\
\text { Pr or i-Pr) phosphonothiolates and corresponding alkylated or protonated salts }\end{array}$ & CR0003 & -- & CWC 1 A3 & & WA ML7 b.1.c \\
\hline Nerve agents & VX: O-Ethyl S-2-diisopropylaminoethyl methyl phosphonothiolate & $50782-69-9$ & $\underline{39793}$ & CWC 1 A3 Example 1 & & $\begin{array}{l}\text { WA ML7 b.1.c Example } \\
1\end{array}$ \\
\hline Sulfur mustards & 2-Chloroethylchloromethylsulfide & $2625-76-5$ & 14557887 & CWC 1A4-1 & & WA ML7 b.2.a.1 \\
\hline Sulfur mustards & Mustard gas: Bis(2-chloroethyl)sulfide & $505-60-2$ & 10461 & CWC 1A4-2 & & WA ML7 b.2.a.2 \\
\hline Sulfur mustards & Bis(2-chloroethylthio)methane & 63869-13-6 & $\underline{522107}$ & CWC 1A4-3 & & WA ML7 b.2.a.3 \\
\hline Sulfur mustards & Sesquimustard: 1,2-Bis(2-chloroethylthio)ethane & $3563-36-8$ & $\underline{19092}$ & CWC 1A4-4 & & WA ML7 b.2.a.4 \\
\hline Sulfur mustards & 1,3-Bis(2-chloroethylthio)-n-propane & $63905-10-2$ & $\underline{522108}$ & CWC 1A4-5 & & WA ML7 b.2.a.5 \\
\hline Sulfur mustards & 1,4-Bis(2-chloroethylthio)-n-butane & 142868-93-7 & $\underline{518918}$ & CWC 1A4-6 & & WA ML7 b.2.a.6 \\
\hline Sulfur mustards & 1,5-Bis(2-chloroethylthio)-n-pentane & $142868-94-8$ & $\underline{22608774}$ & CWC 1A4-7 & & WA ML7 b.2.a.7 \\
\hline Sulfur mustards & Bis(2-chloroethylthiomethyl)ether & 63918-90-1 & $\underline{22608780}$ & CWC 1A4-8 & & WA ML7 b.2.a.8 \\
\hline Sulfur mustards & O-Mustard: Bis(2-chloroethylthioethyl)ether & $63918-89-8$ & 45452 & CWC 1A4-9 & & WA ML7 b.2.a.9 \\
\hline Lewisites & Lewisite 1: 2-Chlorovinyldichloroarsine & $541-25-3$ & $\underline{5372798}$ & CWC 1A5-1 & & WA ML7 b.2.b.1 \\
\hline Lewisites & Lewisite 2: Bis(2-chlorovinyl)chloroarsine & 40334-69-8 & $\underline{568106}$ & CWC 1A5-2 & & WA ML7 b.2.b. 2 \\
\hline Lewisites & Lewisite 3: Tris(2-chlorovinyl)arsine & 40334-70-1 & $\underline{5352143}$ & CWC 1A5-3 & & WA ML7 b.2.b.3 \\
\hline Nitrogen mustards & HN1: Bis(2-chloroethyl)ethylamine & $538-07-8$ & $\underline{10848}$ & CWC 1A6-1 & & WA ML7 b.2.c. 1 \\
\hline Nitrogen mustards & HN2: Bis(2-chloroethyl)methylamine & $51-75-2$ & $\underline{4033}$ & CWC 1A6-2 & & WA ML7 b.2.c. 2 \\
\hline Nitrogen mustards & HN3: Tris(2-chloroethyl)amine & $555-77-1$ & $\underline{5561}$ & CWC 1A6-3 & & WA ML7 b.2.c. 3 \\
\hline Toxins & Saxitoxin & $35523-89-8$ & 56947150 & CWC $1 \mathrm{~A} 7$ & & \\
\hline Toxins & Ricin & $9009-86-3$ & P02879 & CWC 1 A8 & & \\
\hline Novichok agents & $\begin{array}{l}\text { P-alkyl }(\mathrm{H} \text { or }<=\mathrm{C} 10 \text {, incl. cycloalkyl) } \mathrm{N}-(1-(\text { dialkyl }(<=\mathrm{C} 10 \text {, incl. cycloalkyl }) \text { amino }) \text { ) alkylidene }(\mathrm{H} \text { or } \\
<=\mathrm{C} 10 \text {, incl. cycloalkyl) phosphonamidic fluorides and corresponding alkylated or protonated salts }\end{array}$ & CR0004 & -- & CWC $1 \mathrm{~A} 13$ & & \\
\hline Novichok agents & $\mathrm{N}$-(1-(di-n-decylamino)-n-decylidene)-P-decylphosphonamidic fluoride & 2387495-99-8 & CID Not Assigned & CWC $1 \mathrm{~A} 13$ Example 1 & & \\
\hline Novichok agents & Methyl-(1-(diethylamino)ethylidene)phosphonamidofluoridate & $2387496-12-8$ & $\underline{132472359}$ & CWC 1 A13 Example 2 & & \\
\hline Novichok agents & $\begin{array}{l}\text { O-alkyl ( } \mathrm{H} \text { or }<=\mathrm{C} 10 \text {, incl. cycloalkyl) } \mathrm{N}-(1-(\text { dialkyl }(<=\mathrm{C} 10 \text {, incl. cycloalkyl ) amino)) alkylidene }(\mathrm{H} \text { or } \\
<=\mathrm{C} 10, \text { incl. cycloalkyl) phosphonamidofluoridates and Corresponding alkylated or protonated } \\
\text { salts }\end{array}$ & CR0005 & --- & CWC $1 \mathrm{~A} 14$ & & \\
\hline Novichok agents & O-n-Decyl N-(1-(di-n-decylamino)-n-decylidene)phosphoramidofluoridate & $2387496-00-4$ & CID Not Assigned & CWC 1A14 Example 1 & & \\
\hline Novichok agents & Methyl-(1-(diethylamino)ethylidene)phosphoramidofluoridate & 2387496-04-8 & 132472360 & CWC 1 A14 Example 2 & & \\
\hline Novichok agents & Ethyl-(1-(diethylamino)ethylidene)phosphoramidofluoridate & $2387496-06-0$ & $\underline{139033607}$ & CWC 1A14 Example 3 & & \\
\hline Novichok agents & Methyl-(bis(diethylamino)methylene)phosphonamidofluoridate & 2387496-14-0 & 146586254 & CWC $1 \mathrm{~A} 15$ & & \\
\hline $\begin{array}{l}\text { Carbamates } \\
\text { (quaternaries of } \\
\text { dimethylcarbamoyloxyp } \\
\text { yridines) }\end{array}$ & $\begin{array}{l}\text { 1-[N,N-dialkyl (<=C10)-N-(n-(hydroxyl, cyano, acetoxy) alkyl (<=C10)) ammonio]-n-[N-(3- } \\
\text { dimethylcarbamoxy-a-picolinyl)-N,N-dialkyl( } \leq \mathrm{C} 10) \text { ammonio]decane dibromide }(\mathrm{n}=1-8)\end{array}$ & CR0006 & --- & CWC 1A16-1 & & \\
\hline $\begin{array}{l}\text { Carbamates } \\
\text { (quaternaries of } \\
\text { dimethylcarbamoyloxyp } \\
\text { yridines) }\end{array}$ & $\begin{array}{l}\text { 1-[N,N-dimethyl-N-(2-hydroxy)ethylammonio]-10-[N-(3-dimethylcarbamoxy- } \alpha \text {-picolinyl)-N,N- } \\
\text { dimethylammonio]decane dibromide }\end{array}$ & $77104-62-2$ & CID Not Assigned & $\begin{array}{l}\text { CWC 1A16-1 Example } \\
1\end{array}$ & & \\
\hline $\begin{array}{l}\text { Carbamates } \\
\text { (quaternaries of } \\
\text { dimethylcarbamoyloxyp } \\
\text { yridines) }\end{array}$ & $\begin{array}{l}\text { 1,n-Bis[N-(3-dimethylcarbamoxy-a-picolyl)-N,N-dialkyl(( } \mathrm{CC} 10) \text { ammonio]-alkane- }(2,(\mathrm{n}-1) \text {-dione }) \\
\text { dibromide (n=2-12) }\end{array}$ & CR0007 & --- & CWC 1A16-2 & & \\
\hline $\begin{array}{l}\text { Carbamates } \\
\text { (quaternaries of } \\
\text { dimethylcarbamoyloxyp } \\
\text { yridines) }\end{array}$ & $\begin{array}{l}\text { 1,10-Bis[N-(3-dimethylcarbamoxy-a-picolyl)-N-ethyl-N-methylammonio]decane-2,9- dione } \\
\text { dibromide }\end{array}$ & $77104-00-8$ & $\underline{\text { CID Not Assigned }}$ & $\begin{array}{l}\text { CWC 1A16-2 Example } \\
1\end{array}$ & & \\
\hline Precursor & Alkyl (Me, Et, n-Pr or i-Pr) phosphonyldifluorides & CR0008 & --- & CWC 1B9 & & WA ML7 c. 1 \\
\hline Precursor & DF: Methylphosphonyldifluoride (AG: Methylphosphonyl difluoride (DF)) & $676-99-3$ & $\underline{69610}$ & CWC 1B9 Example 1 & AG 4 & WA ML7 c.1 Example 1 \\
\hline Precursor & Ethylphosphonyl difluoride & $753-98-0$ & $\underline{12585494}$ & $\begin{array}{l}\text { Not listed individually } \\
\text { but member of family } \\
\text { CWC } 1 \text { B9 }\end{array}$ & AG 23 & $\begin{array}{l}\text { Not listed individually } \\
\text { but member of family } \\
\text { WA ML7 c. } 1\end{array}$ \\
\hline Precursor & $\begin{array}{l}\text { O-Alkyl (H or }<=C 10 \text {, incl. cycloalkyl) O-2-dalkyl (Me, Et, n-Pr or i-Pr)-aminoethyl alkyl (Me, Et, n- } \\
\text { Pr or i-Pr) phosphonites and corresponding alkylated or protonated salts }\end{array}$ & CR0009 & --- & CWC 1B10 & & WA ML7 c.2 \\
\hline Precursor & QL: O-Ethyl O-2-diisopropylaminoethyl methylphosphonite & $57856-11-8$ & $\underline{170325}$ & CWC 1B10 Example 1 & AG 29 & WA ML7 c. 2 Example 1 \\
\hline Precursor & Chlorosarin: O-Isopropyl methylphosphonochloridate & $1445-76-7$ & 102124 & CWC 1B11 & & WA ML7 c. 3 \\
\hline Precursor & Chlorosoman: O-Pinacolyl methylphosphonochloridate & $7040-57-5$ & 145983 & CWC 1B12 & & WA ML7 c.4 \\
\hline Nerve agents & Amiton: O,O-Diethyl S-[2-(diethylamino)ethyl] phosphorothiolate & $78-53-5$ & $\underline{6542}$ & CWC 2A1 & & \\
\hline Choking agents & PFIB: 1,1,3,3,3-Pentafluoro-2-(trifluoromethyl)-1-propene & $382-21-8$ & $\underline{61109}$ & CWC 2A2 & & \\
\hline Central incapacitants & BZ: 3-Quinuclidinyl benzilate $\left(^{*}\right)$ & $6581-06-2$ & $\underline{23056}$ & CWC 2 A3 & & WA ML7 b.3.a \\
\hline Precursor & $\begin{array}{l}\text { Chemicals, except for those listed in Schedule 1, containing a phosphorus atom to which is } \\
\text { bonded one methyl, ethyl or propyl (normal or iso) group but not further carbon atoms, }\end{array}$ & CR0010 & -- & CWC 2B4 & & \\
\hline Precursor & Methylphosphonyl dichloride (AG: Methylphosphonyl dichloride (DC)) & $676-97-1$ & $\underline{12671}$ & CWC 2B4 Example 1 & AG 5 & \\
\hline Precursor & Dimethyl methylphosphonate & $756-79-6$ & 12958 & CWC 2B4 Example 2 & AG 3 & \\
\hline Precursor & O-Ethyl S-phenyl ethylphosphonothiolothionate & $944-22-9$ & $\underline{13676}$ & CWC 2B4 Exception 1 & & \\
\hline Precursor & Diethyl ethylphosphonate & 78-38-6 & $\underline{6534}$ & $\begin{array}{l}\text { Not listed individually } \\
\text { but member of family } \\
\text { CWC 2B4 }\end{array}$ & AG 17 & \\
\hline Precursor & Ethylphosphinyl dichloride & $1498-40-4$ & 15157 & $\begin{array}{l}\text { Not listed individually } \\
\text { but member of family } \\
\text { CWC 2B4 }\end{array}$ & AG 21 & \\
\hline Precursor & Ethylphosphonyl dichloride & $1066-50-8$ & $\underline{70596}$ & $\begin{array}{l}\text { Not listed individually } \\
\text { but member of family } \\
\text { CWC 2B4 }\end{array}$ & AG 22 & \\
\hline
\end{tabular}




\begin{tabular}{|c|c|c|c|c|c|}
\hline Precursor & Methylphosphinyl dichloride & $676-83-5$ & $\underline{61194}$ & $\begin{array}{l}\text { Not IIsted individually } \\
\text { but member of family } \\
\text { CWC 2B4 }\end{array}$ & AG 26 \\
\hline Precursor & Diethyl methylphosphonite & $15715-41-0$ & $\underline{85063}$ & $\begin{array}{l}\text { Not listed individually } \\
\text { but member of family } \\
\text { CWC 2B4 }\end{array}$ & AG 33 \\
\hline Precursor & Dimethyl ethylphosphonate & 6163-75-3 & $\underline{521983}$ & $\begin{array}{l}\text { Not listed individually } \\
\text { but member of family } \\
\text { CWC 2B4 }\end{array}$ & AG 34 \\
\hline Precursor & Ethylphosphinyl difluoride & $430-78-4$ & $\underline{23280220}$ & $\begin{array}{l}\text { Not listed individually } \\
\text { but member of family } \\
\text { CWC 2B4 }\end{array}$ & AG 35 \\
\hline Precursor & Methylphosphinyl difluoride & $753-59-3$ & 136565 & $\begin{array}{l}\text { Not listed individually } \\
\text { but member of family } \\
\text { CWC 2B4 }\end{array}$ & AG 36 \\
\hline Precursor & Methylphosphonic acid & $993-13-5$ & $\underline{13818}$ & $\begin{array}{l}\text { Not listed individually } \\
\text { but member of family } \\
\text { CWC 2B4 }\end{array}$ & AG 55 \\
\hline Precursor & Diethyl methylphosphonate & $683-08-9$ & $\underline{12685}$ & $\begin{array}{l}\text { Not listed individually } \\
\text { but member of family } \\
\text { CWC 2B4 }\end{array}$ & AG 56 \\
\hline Precursor & Methylphosphonothioic dichloride & $676-98-2$ & $\underline{61195}$ & $\begin{array}{l}\text { Not listed individually } \\
\text { but member of family } \\
\text { CWC 2B4 }\end{array}$ & AG 63 \\
\hline Precursor & N,N-Dialkyl (Me, Et, n-Pr or i-Pr) phosphoramidic dihalides & CR00011 & --- & CWC 2B5 & \\
\hline Precursor & $\mathrm{N}, \mathrm{N}$-Dimethylaminophosphoryl dichloride & $677-43-0$ & 12673 & $\begin{array}{l}\text { Not listed individually } \\
\text { but member of family } \\
\text { CWC 2B5 }\end{array}$ & AG 57 \\
\hline Precursor & Dialkyl (Me, Et, n-Pr or i-Pr) N,N-dialkyl (Me, Et, n-Pr or i-Pr)-phosphoramidates & CR00012 & -- & CWC 2B6 & \\
\hline Precursor & Diethyl N,N-dimethylphosphoramidate & 2404-03-7 & $\underline{520087}$ & $\begin{array}{l}\text { Not listed individually } \\
\text { but member of family } \\
\text { CWC 2B6 }\end{array}$ & AG 18 \\
\hline Precursor & Arsenic trichloride & $7784-34-1$ & $\underline{24570}$ & CWC 2B7 & AG 31 \\
\hline Precursor & 2,2-Diphenyl-2-hydroxyacetic acid (AG: Benzilic Acid) & 76-93-7 & $\overline{6463}$ & CWC 2B8 & AG 32 \\
\hline Precursor & Quinuclidin-3-ol & 1619-34-7 & 15381 & CWC 2B9 & AG 13 \\
\hline Precursor & $\mathrm{N}, \mathrm{N}$-Dialkyl (Me, Et, n-Pr or i-Pr) aminoethyl-2-chlorides and corresponding protonated salts & CR00013 & -- & CWC 2B10 & \\
\hline Precursor & N,N-Diisopropyl-(beta)-aminoethyl chloride & $96-79-7$ & $\underline{7312}$ & $\begin{array}{l}\text { Not listed individually } \\
\text { but member of family } \\
\text { CWC } 2 \text { B } 10\end{array}$ & AG 11 \\
\hline Precursor & $\mathrm{N}, \mathrm{N}$-Diisopropyl-2-aminoethyl chloride hydrochloride & $4261-68-1$ & $\underline{77942}$ & $\begin{array}{l}\text { Not listed individually } \\
\text { but member of family } \\
\text { CWC } 2 \text { B } 10\end{array}$ & AG 54 \\
\hline Precursor & N,N-Dialkyl (Me, Et, n-Pr or i-Pr) aminoethane-2-ols and corresponding protonated salts & CR00014 & -.- & CWC 2B11 & \\
\hline Precursor & $\mathrm{N}, \mathrm{N}$-Dimethylaminoethanol and corresponding protonated salts & $108-01-0$ & 7902 & CWC 2B11 Exception 1 & \\
\hline Precursor & $\mathrm{N}, \mathrm{N}$-Diethylaminoethanol and corresponding protonated salts (AG: Diethylaminoethanol) & $100-37-8$ & 7497 & CWC 2B11 Exception 2 & AG 49 \\
\hline Precursor & N,N-Diisopropyl-(beta)-amino-ethanol & $96-80-0$ & 7313 & $\begin{array}{l}\text { Not listed individually } \\
\text { but member of family } \\
\text { CWC 2B11 }\end{array}$ & AG 27 \\
\hline Precursor & $\mathrm{N}, \mathrm{N}$-Dialkyl (Me, Et, n-Pr or i-Pr) aminoethane-2-thiols and corresponding protonated salts & CR00015 & -.- & CWC 2B12 & \\
\hline Precursor & $\mathrm{N}, \mathrm{N}$-Diisopropyl-(beta)-aminoethane thiol & $5842-07-9$ & $\underline{111102}$ & $\begin{array}{l}\text { Not listed individually } \\
\text { but member of family } \\
\text { CWC } 2 \text { B } 12\end{array}$ & AG 12 \\
\hline Precursor & $\mathrm{N}, \mathrm{N}$-Diisopropylaminoethanethiol hydrochloride & $41480-75-5$ & 3084829 & $\begin{array}{l}\text { Not listed individually } \\
\text { but member of family } \\
\text { CWC } 2 \text { B12 }\end{array}$ & AG 65 \\
\hline Precursor & Thiodiglycol: Bis(2-hydroxyethyl)sulfide (AG: Thiodiglycol) & $111-48-8$ & $\underline{5447}$ & CWC $2 \mathrm{~B} 13$ & AG 1 \\
\hline Precursor & Pinacolyl alcohol: 3,3-Dimethylbutan-2-ol (AG: Pinacolyl alcohol) & $464-07-3$ & $\overline{10045}$ & CWC 2B14 & AG 28 \\
\hline Choking agents & Phosgene: Carbonyl dichloride & $75-44-5$ & $\underline{6371}$ & CWC 3A1 & \\
\hline Blood agents & Cyanogen chloride & $506-77-4$ & $\overline{10477}$ & CWC $3 \mathrm{~A} 2$ & \\
\hline Blood agents & Hydrogen cyanide & 74-90-8 & $\underline{768}$ & CWC 3 A 3 & \\
\hline Choking agents & Chloropicrin: Trichloronitromethane & $76-06-2$ & $\underline{6423}$ & CWC $3 A 4$ & \\
\hline Precursor & Phosphorus oxychloride & $10025-87-3$ & $\underline{24813}$ & CWC 3B5 & AG 2 \\
\hline Precursor & Phosphorus trichloride & $7719-12-2$ & $\underline{24387}$ & CWC 3B6 & AG 7 \\
\hline Precursor & Phosphorus pentachloride & $10026-13-8$ & $\underline{24819}$ & CWC 3B7 & AG 38 \\
\hline Precursor & Trimethyl phosphite & $121-45-9$ & $\overline{8472}$ & CWC 3B8 & AG 8 \\
\hline Precursor & Triethyl phosphite & $122-52-1$ & 31215 & CWC 3B9 & AG 30 \\
\hline Precursor & Dimethyl phosphite & $868-85-9$ & 6327114 & CWC 3B10 & AG 6 \\
\hline Precursor & Diethyl phosphite & $762-04-9$ & $\overline{6327654}$ & CWC 3B11 & AG 19 \\
\hline Precursor & Sulfur monochloride & $10025-67-9$ & 24807 & CWC 3B12 & AG 51 \\
\hline Precursor & Sulfur dichloride & $10545-99-0$ & $\overline{25353}$ & CWC 3B13 & AG 52 \\
\hline Precursor & Thionyl chloride & $7719-09-7$ & $\overline{24386}$ & CWC 3B14 & AG 9 \\
\hline Precursor & Ethyldiethanolamine & $139-87-7$ & 8769 & CWC 3B15 & AG 59 \\
\hline Precursor & Methyldiethanolamine & $105-59-9$ & $\overline{7767}$ & CWC 3B16 & \\
\hline Precursor & Triethanolamine & $102-71-6$ & $\overline{7618}$ & CWC 3B17 & AG 46 \\
\hline Precursor & 3-Hydroxy-1-methylpiperidine & $3554-74-3$ & 98016 & & AG 10 \\
\hline Precursor & Potassium fluoride & $7789-23-3$ & $\underline{\underline{522689}}$ & & AG 14 \\
\hline Precursor & 2-Chloroethanol & $107-07-3$ & 34 & & AG 15 \\
\hline Precursor & Dimethylamine & $124-40-3$ & 674 & & AG 16 \\
\hline Precursor & Dimethylamine hydrochloride & $506-59-2$ & $\overline{10473}$ & & AG 20 \\
\hline Precursor & Hydrogen fluoride & $7664-39-3$ & $\overline{14917}$ & & AG 24 \\
\hline Precursor & Methyl benzilate & $76-89-1$ & $\overline{66159}$ & & AG 25 \\
\hline Precursor & 3-Quinuclidone & $3731-38-2$ & 19507 & & AG 37 \\
\hline Precursor & Pinacolone & $75-97-8$ & 6416 & & AG 39 \\
\hline Precursor & Potassium cyanide & $151-50-8$ & $\underline{9032}$ & & AG 40 \\
\hline Precursor & Potassium bifluoride & $7789-29-9$ & 11829350 & & AG 41 \\
\hline Precursor & Ammonium bifluoride & $1341-49-7$ & 14935 & & AG 42 \\
\hline Precursor & Sodium bifluoride & $1333-83-1$ & $\overline{219061}$ & & AG 43 \\
\hline Precursor & Sodium fluoride & $7681-49-4$ & $\underline{5235}$ & & AG 44 \\
\hline Precursor & Sodium cyanide & $143-33-9$ & $\overline{8929}$ & & AG 45 \\
\hline Precursor & Phosphorus pentasulphide & $1314-80-3$ & 14817 & & AG 47 \\
\hline Precursor & Diisopropylamine & $108-18-9$ & 7912 & & AG 48 \\
\hline Precursor & Sodium sulphide & $1313-82-2$ & $\underline{237873}$ & & AG 50 \\
\hline Precursor & Triethanolamine hydrochloride & $637-39-8$ & 101814 & & AG 53 \\
\hline Precursor & Triisopropyl phosphite & $116-17-6$ & 8304 & & AG 58 \\
\hline Precursor & O,O-Diethyl phosphorothioate & $2465-65-8$ & $\underline{655}$ & & AG 60 \\
\hline Precursor & O,O-Diethyl phosphorodithioate & $298-06-6$ & $\underline{9274}$ & & AG 61 \\
\hline Precursor & Sodium hexafluorosilicate & $16893-85-9$ & 28127 & & AG 62 \\
\hline Precursor & Diethylamine & 109-89-7 & 8021 & & AG 64 \\
\hline Precursor & Methyl dichlorophosphate & $677-24-7$ & $\overline{69611}$ & & AG 66 \\
\hline Precursor & Ethyl Dichlorophosphate & $1498-51-7$ & 15158 & & AG 67 \\
\hline Precursor & Methyl Difluorophosphate & $22382-13-4$ & $\overline{123401}$ & & AG 68 \\
\hline Precursor & Ethyl Difluorophosphate & $460-52-6$ & $\overline{120250}$ & & AG 69 \\
\hline Precursor & Diethyl Chlorophosphite & $589-57-1$ & $\underline{68530}$ & & AG 70 \\
\hline Draniuronr & Mathil Chinmfli inmenhnon & 751_n1 \& & 210 & & \\
\hline
\end{tabular}




\begin{tabular}{|c|c|c|c|c|c|}
\hline & 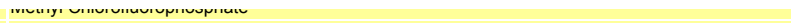 & . & & & \\
\hline Precursor & Ethyl Chlorofluorophosphate & $762-77-6$ & $\underline{23416048}$ & AG 72 & \\
\hline Precursor & $\mathrm{N}, \mathrm{N}$-Dimethylformamidine & $44205-42-7$ & 12669390 & AG 73 & \\
\hline Precursor & $\mathrm{N}, \mathrm{N}$-Diethylformamidine & $90324-67-7$ & 17912027 & AG 74 & \\
\hline Precursor & $\mathrm{N}, \mathrm{N}$-Dipropylformamidine & $48044-20-8$ & $\underline{87832225}$ & AG 75 & \\
\hline Precursor & $\mathrm{N}, \mathrm{N}$-Diisopropylfromamidine & $857522-08-8$ & 19782034 & AG 76 & \\
\hline Precursor & $\mathrm{N}, \mathrm{N}$-Dimethylacetamidine & $2909-14-0$ & $\underline{6394935}$ & AG 77 & \\
\hline Precursor & $\mathrm{N}, \mathrm{N}$-Diethylacetamidine & $14277-06-6$ & 4243241 & AG 78 & \\
\hline Precursor & $\mathrm{N}, \mathrm{N}$-Dipropylacetamidine & $1339586-99-0$ & $\underline{62047941}$ & AG 79 & \\
\hline Precursor & $\mathrm{N}, \mathrm{N}$-Dimethylpropanamidine & $56776-14-8$ & $\underline{12375523}$ & AG 80 & \\
\hline Precursor & $\mathrm{N}, \mathrm{N}$-Diethylpropanamidine & 84764-73-8 & 13109962 & AG 81 & \\
\hline Precursor & $\mathrm{N}, \mathrm{N}$-Dipropylpropanamidine & $1341496-89-6$ & $\underline{62047942}$ & AG 82 & \\
\hline Precursor & $\mathrm{N}, \mathrm{N}$-Dimethylbutanamidine & $1340437-35-5$ & 17928752 & AG 83 & \\
\hline Precursor & $\mathrm{N}, \mathrm{N}$-Diethylbutanamidine & $53510-30-8$ & $\underline{57106491}$ & AG 84 & \\
\hline Precursor & $\mathrm{N}, \mathrm{N}$-Dipropylbutanamidine & $1342422-35-8$ & $\underline{62048118}$ & AG 85 & \\
\hline Precursor & $\mathrm{N}, \mathrm{N}$-Diisopropylbutanamidine & $1315467-17-4$ & $\underline{101801650}$ & AG 86 & \\
\hline Precursor & $\mathrm{N}, \mathrm{N}$-Dimethylisobutanamidine & $321881-25-8$ & $\underline{21623186}$ & AG 87 & \\
\hline Defoliants & Butyl 2-chloro-4-fluorophenoxyacetate (LNF) & $1692-85-9$ & $\underline{54941708}$ & & WA ML7 b.4.a \\
\hline Defoliants & $\begin{array}{l}\text { 2,4,5-trichlorophenoxyacetic acid mixed with 2,4-dichlorophenoxyacetic acid (Agent Orange) } \\
\text { Note: this is the entry name given in ML7 }\end{array}$ & $\begin{array}{l}8015-35-8 \\
\text { Note: this CAS Registry } \\
\text { Number is not given in } \\
\text { ML7 but can be inferred } \\
\text { from the name given in } \\
\text { ML7 }\end{array}$ & $\frac{24683}{1}$ & & $\begin{array}{l}\text { WA ML7 b.4.b Free } \\
\text { Acid Form }\end{array}$ \\
\hline Defoliants & $\begin{array}{l}\text { 2,4,5-trichlorophenoxyacetic acid butyl ester mixed with 2,4-dichlorophenoxyacetic acid butyl ester } \\
\text { (Agent Orange) } \\
\text { Note: this name is not given in ML7 but can inferred from the CAS Registry Number given in ML7 } \\
(39277-47-9)\end{array}$ & $\begin{array}{l}39277-47-9 \\
\text { Note: this is the entry } \\
\text { CAS Registry Number } \\
\text { given in ML7 }\end{array}$ & 38264 & & $\begin{array}{l}\text { WA ML7 b.4.b Butyl } \\
\text { Ester Form }\end{array}$ \\
\hline Defoliants & 2,4,5-trichlorophenoxyacetic acid & $93-76-5$ & $\underline{1480}$ & & $\begin{array}{l}\text { WA ML7 b.4.b } \\
\text { Component } 1\end{array}$ \\
\hline Defoliants & 2,4-dichlorophenoxyacetic acid & $94-75-7$ & $\underline{1486}$ & & $\begin{array}{l}\text { WA ML7 b.4.b } \\
\text { Component } 2\end{array}$ \\
\hline Riot control agents & $\alpha$-Bromobenzeneacetonitrile, (Bromobenzyl cyanide) (CA) & $5798-79-8$ & $\underline{22044}$ & & WA ML7 d.1 \\
\hline Riot control agents & [(2-chlorophenyl) methylene] propanedinitrile, (o-Chlorobenzylidenemalononitrile) (CS) & 2698-41-1 & 17604 & & WA ML7 d.2 \\
\hline Riot control agents & 2-Chloro-1-phenylethanone, Phenylacyl chloride ( $\omega$-chloroacetophenone) (CN) & $532-27-4$ & 10757 & & WA ML7 d.3 \\
\hline Riot control agents & Dibenz-(b,f)-1,4-oxazephine, (CR) & $257-07-8$ & --- & & WA ML7 $\mathrm{d} .4$ \\
\hline Riot control agents & 10-Chloro-5,10-dihydrophenarsazine, (Phenarsazine chloride), (Adamsite), (DM) & $578-94-9$ & 11362 & & WA ML7 d.5 \\
\hline Riot control agents & N-Nonanoylmorpholine, (MPA) & $5299-64-9$ & $\underline{79182}$ & & WA ML7 d.6 \\
\hline
\end{tabular}

